\title{
A Note on the Cryostatic Stability of Superconducting Composites
}

\author{
Wilhelm F. Gauster
}

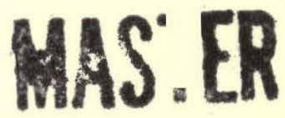




\section{DISCLAIMER}

This report was prepared as an account of work sponsored by an agency of the United States Government. Neither the United States Government nor any agency Thereof, nor any of their employees, makes any warranty, express or implied, or assumes any legal liability or responsibility for the accuracy, completeness, or usefulness of any information, apparatus, product, or process disclosed, or represents that its use would not infringe privately owned rights. Reference herein to any specific commercial product, process, or service by trade name, trademark, manufacturer, or otherwise does not necessarily constitute or imply its endorsement, recommendation, or favoring by the United States Government or any agency thereof. The views and opinions of authors expressed herein do not necessarily state or reflect those of the United States Government or any agency thereof. 


\section{DISCLAIMER}

Portions of this document may be illegible in electronic image products. Images are produced from the best available original document. 


\begin{tabular}{|l|}
\hline $\begin{array}{c}\text { Printed in the United States of America. Available from } \\
\text { National Technical Information Service } \\
\text { U.S. Department of Commerce }\end{array}$ \\
$\begin{array}{l}5285 \text { Port Royal Road, Springfield, Virginia } 22161 \\
\text { Price: Printed Copy } \$ \text {; Microfiche } \$ 3.00\end{array}$ \\
$\begin{array}{l}\text { This report was prepared as an account of work sponsored by an agency of the United } \\
\text { States Government. Neither the United States Government nor any agency thereof, nor } \\
\text { any of their employees, contractors, subcontractors, or their employees, makes any } \\
\text { warranty, express or implied, nor assumes any legal liability or responsibility for any } \\
\text { third party's use or the results of such use of any information, apparatus, product or } \\
\text { process disclosed in this report, nor represents that its use by such third party would } \\
\text { not infringe privately owned rights. }\end{array}$ \\
\hline
\end{tabular}


Contract No. W-7405-eng-26

FUSION ENERGY DIVISION

\title{
A NOTE ON THE CRYOSTATIC STABILITY OF
}

SUPERCONDUCTING COMPOSITES

(with special attention to the work of B. J. MADDOCK et al.)

\author{
Wilhelm F. Gauster
}

(Short-term consultant)

Date Pub1ished - February 1978

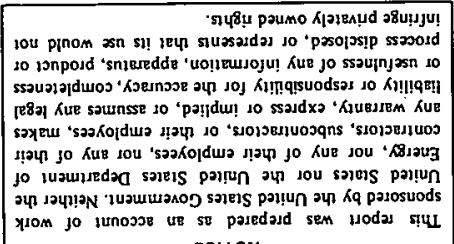

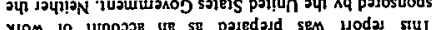

ZIIION

NOTICE This document contains information of a preliminary nature. It is subject to revision or correction and therefore does not represent a final report.

\author{
OAK RIDGE NATIONAL LABORATORY \\ nak Ridge, Tennessee 37830 \\ operated by \\ UNION CARBIDE CORPORATION \\ for the \\ DEPARTMENT OF ENERGY
}


THIS PAGE

\section{WAS INTENTIONALLY \\ LEFT BLANK}


TABLE OF CONTENTS

Abstract . . . . . . . . . . . . . . . . . . . . 1

1 Introduction . . . . . . . . . . . . . . . . . 2

2 Basic Concepts . . . . . . . . . . . . . . . . . . 3

2.1 Zero-dimensional analysis . . . . . . . . . . . . 3

2.1.1 Basic equations . . . . . . . . . . . . . 3

2.1.2 $g(\tau, i)$ and $q(\tau)$ diagrams, and currentvoltage characteristics............ 6

2.2 One-dimensional analysis . . . . . . . . . . . 9 9

2.2.1 Basic equations .. . . . . . . . . . . . 9

2.2.2 Normalized differential equations for the ranges $\mathrm{A}, \mathrm{B}$ and $\mathrm{C}$. . . . . . . . . . 10

2.2.3 $\tau(x)$ characteristic for the cold end recovery current . . . . . . . . . . . . 11

2.2.4 Numerical example for the temperature
profile $\tau(\mathrm{x})$ for cold end recovery ....... 13

3 The Equal Area Condition by Maddock et al. . . . . . . . . . . 14

3.1 The differential equation $y^{\prime \prime}=F(y)$ and certain kinds of stability criteria . . . . . . . . . 14

3.2 Derivation of the Equal Area Condition . . . . . . . . 16

3.3 Application of the Equal Area Condition to the case of "Cold End Recovery" . . . . . . . . . . . 18

3.4 Performance of a resistance wire with cooling by nucleate and by film boiling helium . . . . . . . . . 19

3.4.1 $\mathrm{Q}(\Delta \mathrm{T})$ and $\Delta \mathrm{T}(\mathrm{x})$ diagrams . . . . . . . . . . . 19

3.4.2 Analytical solution for the transition value of the Joule heating rate G . . . . . . . 21

3.4.3 Check of the Equal Area Condition . . . . . . . . 23

4 Discussion of the presuppositions for the validity of the Equal Area Condition . . . . . . . . . . . . . . . . 23

4.1 The "long" wire . . . . . . . . . . . . . . . . 23

4.1.1 Acceptable temperature tolerances. $\Delta \mathrm{T}(\mathrm{x})$ vs $\mathrm{Q}(\Delta \mathrm{T})$ diagrams . . . . . . . . . . 23

4.1.2 The condition IimS $=0$. Integration over limits located inside the wire length . . . . . . 26

4.2 One-dimensional equilibrium states of a liquid helium cooled resistance wire considering finite lengths of the nucleate and of the $f i l m$ bolling zones . . . . . . . 27 
4.2.1 Basic equations. The $G\left(\Delta \mathrm{T}_{\mathrm{M}}\right)$ diagram . . . . . . 27

4.2.2 Numerical examples of $G(a)$ and $G\left(\Delta T_{M}\right)$

5 One-dimensional analysis of the second kind . . . . . . . . . 31

5.1 Basic equations .. . . . . . . . . . . . . 31

5.1.1 Computer solutions vs analytical solutions . . . . 31

5.1.2 Example of a resistance wire cooled by liquid

helium ( $I$ is a linear function of the time) . . . . 32

5.1:3 Equations for superconducting composites . . . . . 33

5.2 One-dimensional analysis of the second kind

applied to a composite with $\alpha<1.0$. . . . . . . . . . 34

5.3 One-dimensional analysis of the second kind

applied to a composite with $\alpha>1.0$. . . . . . . . . 35

6 Is the equal area condition a sufficient criterion

for cryostatic stabilization? . . . . . . . . . . . . . 38

7 Summary . . . . . . . . . . . . . . . . . 38

Acknowledgement . . . . . . . . . . . . . . . 41

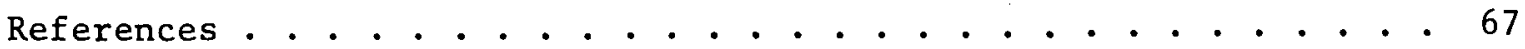




\section{ABSTRACT}

This note contains a careful discussion of the "equal area condition" developed by Maddock et al. In order to make the essential points as clear as possible, I derived analytical solutions under simplifying. assumptions (simple model for heat transfer by nucleate and film boiling. liquid helium; constant heat conduction and specific heat) instead of using more realistic but less controllable computer calculations. A quantitative definition of the concept of a long wire is given. Numerical examples for the Maddock transition characterized by the equal area condition are given for a long superconducting composite with linear cooling and for a liquid helium-cooled resistance wire of finite length. In addition, cases are shown where instead of applying the equal area stability condition, time-dependent solutions should be considered. 


\section{INTRODUCTION}

Some time ago I wrote a "Superconducting Magnet Development Program Note" on the cryostatic stability of superconductors ${ }^{1}$ and restricted myself to the simplest zero-dimensional cases (stationary solutions, and heat flow in the axial direction of the conductor not considered) of the theory of Stekly et al. ${ }^{2}$ This was done at the request of a few colleagues of the Magnetics and Superconductivity Section of the Fusion Energy Division of ORNL, and I myself was somewhat astonished about their interest in such an almost trivial undertaking.

During the discussion of my note, the question was raised whether it would not have been more appropriate to base my elementary presentation not on Stekly's original work but on the approach by B. J. Maddock et al. 3,4 Therefore, I reread these two papers carefully. I recognized again that the equal area condition is an interesting and elegant way to understand (at least semiquantitatively) various important experimental results concerning the stability of superconducting composites and, furthermore, that Maddock's group had command over great experimental experience and - in a good sense of the word - over much physical intuition. However, I had the impression that Maddock himself had the feeling that several points of his presentation needed additional consideration. This seemed to me especially obvious when reading "Appendix 1: Velocity of Propagation" and "Appendix 2: Length of Transition Region" of his paper. I believed that it would be worthwhile to check Maddock's deductions in detail and to discuss carefully in which cases the application of Maddock's ideas would be of advantage.

In talking with my colleagues at the Magnetics and Superconductivity Section, I found not only interest in discussing carefully the applicability of the equal area condition but also it turned out that for quite a few persons everything which could contribute to a clearer understanding of the basic ideas of Maddock et al. would be welcome. Therefore, I am grateful to Marty Lubell for his encouragement and for giving me an opportunity to work out this program note. 


\section{BASIC CONCEPTS}

\subsection{ZERO-DIMENSIONAL ANALYSIS}

\subsubsection{Basic Equations}

Z.J.J. Stekly ${ }^{2}$ introduced the expression "zero-dimensional analysis" for the analysis of the stationary behavior of a thin and long superconducting composite when the axial heat flow is not taken into account. In this case

$G(\Delta T, I)=Q(\Delta T)$,

where $G(\Delta T, I$ ) is the Joule heat (in $W$ ) produced per unit surface area (in $\mathrm{cm}^{2}$ ) of the composite conductor and $Q(\Delta T)$ is the heat flux per unit surface area from the conductor into the coolant. The temperature difference (in $\mathrm{K}$ ) between the wire and the helium bath is given as

$\Delta \mathrm{T}=\mathrm{T}-\mathrm{T}_{\mathrm{b}}$

The current (I) flowing through a superconducting composite wire consists of the superconductor current $\left(\mathrm{I}_{\mathrm{s}}\right)$ and the current $\left(\mathrm{I}_{\mathrm{Cu}}\right)$ which flows through the substrate (frequently but not necessarily copper). For any specified magnetic field $(H)$ below the critical field, the critical superconductor current $\left(I_{c}\right)$ is in good approximation:

$I_{c}=I_{c b}\left(1-\frac{T-T_{b}}{T_{c}-T_{b}}\right)$,

where $I_{c b}=I_{c}\left(T_{b}\right)$. In general, the flux flow resistance of the superconductor $\left(R_{f}\right)$ is much higher than the copper resistance $\left(R_{C u}\right)$, and it can be shown that $I_{s}$ is approximately equal to the critical superconductor current $\left(I_{c}\right)$ at the same temperature $(T)$.

Since the currents through the superconductor and through the substrate are in parallel, the voltage $(V)$ per unit length is 
$V=I_{C u} R_{C u}=\left(I-I_{s}\right) R_{C u}=\left[I-I_{c b}\left(1-\frac{T-T_{b}}{T_{c}-T_{b}}\right)\right] R_{C u} \cdot$

We introduce the following normalized quantities:

$\frac{I}{I_{c b}} \equiv i ; \frac{I_{s}}{I_{c b}} \equiv i_{s} ; \frac{I_{c}}{I_{c b}} \equiv i_{c} ; \frac{I_{C u}}{I_{c b}} \equiv i_{C u} ;$

$\frac{\mathrm{v}}{\mathrm{R}_{\mathrm{Cu}} \mathrm{I}_{c b}} \equiv \mathrm{v}\left(=i_{\mathrm{Cu}}\right) ; \quad \frac{\mathrm{T}-\mathrm{T}_{\mathrm{b}}}{\mathrm{T}_{c}-\mathrm{T}_{\mathrm{b}}} \equiv \tau$.

Equation (4) can be written as

$v=i-i+\tau$,

with $v \geq 0, i \geq 0$, and $\tau \geq 0$

Under the special assumption of a linear heat flux $(Q=h \Delta T)$, Eq. (1) assumes the form

$G=\frac{V I}{P}=Q=h \Delta T$

Introducing Stekly's constant,

$\alpha \equiv \frac{R_{C u} I_{c h}^{2}}{\operatorname{Ph}\left(T_{c}-T_{b}\right)}$.

Equation (7) can be written in the form

$\mathrm{g}=\alpha \mathrm{i} \mathrm{v}=\tau=\mathrm{q}$.

If we consider a nonlinear relation between $Q$ and $\Delta T$, it is still convenient to use a constant $\alpha_{0}$ similar to $\alpha$. Analogous to Eq. (8), the 
constant $\alpha_{0}$ is defined by

$\alpha_{0} \equiv \frac{\mathrm{R}_{\mathrm{Cu}} \mathrm{I}_{\mathrm{cb}}^{2}}{\mathrm{Ph}\left(\mathrm{T}_{\mathrm{c}}-\mathrm{T}_{\mathrm{b}}\right)}$,

where $h_{0}$ is a constant with the dimension $\mathrm{Wcm}^{-2} \mathrm{~K}^{-1}$ and with an arbitrarily chosen magnitude which has nothing to do with the actual function $Q(\Delta T)$.

For instance we can choose $\mathrm{h}_{\mathrm{o}}=1,0 \mathrm{Wcm}^{-2} \mathrm{~K}^{-1}$.

We normalize the quantities $G$ and $Q$ as follows:

$g=\frac{G}{h_{0}\left(T_{c}-T_{b}\right)} \quad$ and $\quad q=\frac{Q}{h_{0}\left(T_{c}-T_{b}\right)}$

In general, i.e., without the assumption $Q=h \Delta T$, we obtain from Eq.

$g(\tau, i)=\alpha_{0} i v=\alpha_{0} i(i-1+\tau)=q(\tau)$

as the normalized zero-dimensional basic equation for the range of current sharing between superconductor and substrate.

From Eq. (12) follow

$i=\sqrt{\left(\frac{1-\tau}{2}\right)^{2}+\frac{\sigma}{\alpha_{0}}}+\left(\frac{1-\tau}{2}\right)$

and

$v=\sqrt{\left(\frac{1-\tau}{2}\right)^{2}+\frac{\sigma}{\alpha_{0}}}-\left(\frac{1-\tau}{2}\right)$.

We can easily check that these equations are not restricted to linear heat flow. From Eq. (10) and (11) follows

$-\frac{\sigma}{\alpha_{0}}-\frac{Q}{h_{o}\left(T_{c}-T_{b}\right)} \cdot \frac{P h_{o}\left(T_{c}-T_{b}\right)}{R_{C u} I_{c b}^{2}}=\frac{Q P}{R_{C u} I_{c b}^{2}}$,

which holds for any linear or nonlinear function $Q(\Delta T)$. 
When the entire current (I) flows through the superconductor, i.e., when $i=i_{s}$ and $i_{C u}=0$, then $g=0$ (Range A). When the entire current flows through the substrate, $i . e$, when $i={ }^{i_{C u}}$ and $i_{s}=0$, then $g=\alpha_{o} i^{2}$ (Range $C$ ). Equation (12) characterizes Range $B$ (current sharing range).

\subsection{2 $g(\tau, i)$ and $g(\tau)$ Diagrams and Current-Voltage Characteristics}

A convenient graphical presentation showing the relations $Q(\Delta T)$ published by Brentari and Smith, by Lyon, and by Butler et al., is Fig. 6 of Ref. 6. That figure uses logarithmic scales. In contrast, Fig. 1 of this note is drawn with linear scales. In addition to the three mentioned diagrams, other kinds of $Q(\Delta T)$ diagrams (from Refs. 3 and 7) are shown. For other nonlinear $\Delta \mathrm{T}-Q$ models employed by Stekly et al., see Ref. 2 .

When looking at Fig. 1, one is impressed by the very great differences between the various characteristics, and it seems that any attempt to achieve at least semiquantitative results might be hopeless. Actually the situation is not quite this bad, because the various characteristics have been obtained under very different experimental conditions. For a series of measurements performed under well defined and well controlled experimental conditions, $a(\Delta T)$ characteristic with acceptable scattering can be expected. For our purpose it is convenient to calculate from the experimental $Q$ values the normalized $q$ values by means of Eq. (11).

Concerning the normalized values of the Joule heat produced, we mentioned before that $g=0$ for Range $A(\tau \leq 1-i)$. For $1-i<\tau<1$, the value of $\mathrm{g}$ can be calculated by means of Eq. (12) (Range B). For $\tau>1$, we previously obtained $g=\alpha_{0} 1^{2}$ (Range $C$ ). These relations hold for $i<1$.

F1gure 2 ohowo $g(\tau)$ diagrams for $\alpha=0.5$, drawn for currents (i) equal to $0.6,1.0,1.2,1 / \sqrt{\alpha}(\doteq 1.414)$, and 1.5 . For any specified value of the parameter $i$, the $g(\tau)$ characteristic is represented by a sequence of two or three pieces of straight lines. For discussing these diagrams it is convenient to compare Fig. 2 with Table 1 . Range A ( $g=0$ ) holds for $0 \leq \tau \leq \tau_{1, v} ; \tau_{1,0}=1-i$. Range $B$ is limited by $g_{\text {min }}$ 
and $g_{\max } \cdot$ For currents $i>1.0$, Range A does not exist and $g_{\min }>0$. For $\tau>1.0$ (Range $C$ ), $g$ has the constant value $g_{\max }=\alpha i^{2}$.

Figure 3 and Table 2 consider $\alpha=1.5$ and currents (i) equal to $0.6,1 / \sqrt{\alpha}(\doteq 0.816), 0.9,1.0$, and 1.1 .

The intersections of the $g(\tau)$ and the $q(\tau)$ characteristics yield the equilibrium states. In Maddock's paper ${ }^{3}$ it is briefly demonstrated that a stable equilibrium can be expected when the $g(\tau)$ curve crosses the $q(\tau)$ curve from above to below it with increasing temperature. A more detailed discussion of stable and unstable equilibrium cases is contained in Ref. 6. Seven possible cases [following from various relative positions of the $g(\tau)$ and $q(\tau)$ characteristics] are shown in Fig. 1 of Ref. 6, and an exact proof by means of a perturbation calculation is given.

In Fig. $2(\alpha=0.5)$ equilibrium states result with $i=0.6$ and $i=1.0$ at $\tau=0$ (see Table 1 ). With $i=1.2$, equilibrium occurs at $\tau=0.3$; with $i=1 / \sqrt{\alpha}(=\sqrt{2})$, equilibrium occurs at $\tau=1.0$; and, finally, for $i=1.5$ it occurs at $\tau=\alpha i^{2}=1.125=g_{\max }$. Each equilibrium point is stable.

For $\alpha=1.5$ (Fig. 3 and Table 2) with $i=0.6$, stable equilibrium occurs for $\tau=0$. When the current is raised to a value just below $1 / \sqrt{\alpha}(\doteq 0.816)$, there is equilibrium at $\tau=0$. As soon as that value of $i$ is just surpassed, equilibrium occurs at $\tau=0$ and $\tau=1.0$. With $i=0.9$, there are three equilibrium states: the first at $\tau=0$, the second at $\tau=0.386$, and the third at $\tau=1.215$. The first and the third are stable; the equilibrium state at 0.386 is unstable. For $i=1.0$, two stable equilibrium states occur with $\tau=0$ and $\tau=1.5$ and, finally, with $i=1.1$ stable equilibrium occurs at $\tau=1.815$.

In the case of zero-dimensional analysis the $\tau(x)$ diagrams are, of course, horizontal straight lines. These $\tau(x)$ diagrams are shown in Figs. 2(a) and $3(a)$.

The normalized voltage per unit length $(v)$ can be obtained from the $g=q$ equilibrium value by dividing it by $\alpha i$ [see Eq. (12)]. In Tables 1 and 2 the $v$ values calculated in this way are shown. In order to draw an $i-v$ characteristic it is necessary to repeat many times the 
procedure of finding the intersections of the $g(\tau)$ with the $q(\tau)$ characteristics for different currents. For an experimental $q(\tau)$ characteristic which cannot be expressed by an analytical expression, this graphical method must be used. If an analytical expression for the $q(\tau)$ characteristic is known, it is much easier to determine the current-voltage characteristic by calculation [see Eqs. (13) and (14)]. Especially for linear cooling, i.e., for $q=\tau$, the calculation is very simple [see Eq. (19) of Ref. 1]:

$v=\frac{i}{i}-\alpha 1$

Figure 4 shows the current-voltage characteristics corresponding to Figs. 2 and 3 and Tables 1 and 2 . These $i-v$ characteristics are reproduced from Fig. 2 of Ref. 1.

As discussed in Ref. 1, current-voltage characteristics show easily whether any specified state of equilibrium is stable or unstable. The same is expressed by Maddock (Ref. 3, p. 266) in the following way: "Usually, superconductors operate in current controlled situations (for example in inductive circuits), and so regions of the current-voltage characteristic with negative differential resistance are unstable and abrupt transitions occur."

It is worthwhile to discuss here the usefulness of the $i-v$ diagrams. We refer to Fig. 4, which shows the current-voltage diagrams for $\alpha=0.5$ and $\alpha=1.5$ (infinitely long wire, axial heat flow not considered). With $\alpha=0.5$, the $i-v$ characteristic is reversible. We assume current regulation and we notice that by increasing $i$ over the value 1.0 we change continuously from Range A to $B$. After reaching the value $i=1 / \sqrt{\alpha}$ $(\doteq 1.414)$, we change again continuously from Range $B$ to Range $C$. When reducing the current ( $i)$, we change continuously from Range $C$ over Range $B$ to Range A.

For $\alpha=1.5$ we expect a jump when $i$ becomes larger than 1.0 and the current changes abruptly from Range A to Range $C$ (which is represented in Fig. 4 by the straight line $v=i$ ) (see Sect. 5). Remaining in Range $C$, the current can be reduced below the value $1 . \dot{0}$. Later we will 
discuss for the case $\alpha>1.0$ certain difficulties of the zero-dimensional analysis, and we will consider the so called "cold end recovery current" which takes the axial heat flow into account.

\subsection{ONE-DIMENSIONAL ANALYSIS}

\subsubsection{Basic Equations}

We consider a thin and long composite superconducting wire in which flux jumps are eliminated by adiabatic or dynamic stabilization. ${ }^{8}$ Our starting point is the well known partial differential equation:

$G(\Delta T, I)=Q(\Delta T)+\frac{A}{P} c(T) \frac{\partial \Delta T}{\partial t}-\frac{\Lambda}{P} \frac{\partial}{\partial x}\left[k(T) \frac{\partial \Delta T}{\partial x}\right]$,

where

A is the cross-section area of the conductor $\left(\mathrm{cm}^{2}\right)$,

$\mathrm{P} \quad$ is the cooled perimeter of the conductor $(\mathrm{cm})$,

$C(T)$ is the specific heat of the composite conductor per unit volume $\left(\mathrm{Jcm}^{-3} \mathrm{~K}^{-1}\right)$,

$\mathrm{k}(\mathrm{T})$ is the thermal conductivity $\left(\mathrm{Wcm}^{-1} \mathrm{~K}^{-1}\right)$,

$G$ is the Joule heat produced per unit surface area of the composite conductor $\left(\mathrm{Wcm}^{-2}\right)$,

Q is the heat flux per unit surface area from the conductor into the coolant $\left(\mathrm{Wcm}^{-2}\right)$, and

$\Delta \mathrm{T}=\mathrm{T}-\mathrm{T}_{\mathrm{b}}$ 1s the temperature difference between the wire and the helium bath.

The current (I) is here a parameter (in contrast to the independent variables $x$ and $t$ and the dependent variable $T$ ). Other parameters (various kinds of external heat sources) which are of great importance will not be discussed in detail in this note.

Stekly et a1. ${ }^{2}$ introduced the expressions "zero-, one-, two-, and three-dimensional analysis." We too used the designation zero-dimensional when both partial differential quotients $\frac{\partial T}{\partial t}$ and $\frac{\partial T}{\partial x}$ were disregarded. However, in contrast to Stekly et al. we will talk about "one-dimensional 
analysis of the first kind" when the partial derivative of second order $\frac{\partial^{2} \mathrm{~T}}{\partial \mathrm{x}^{2}}$ only is taken into account. Furthermore, we will call a discussion "one-dimensional analysis of the second kind" when only the partial differential quotient $\frac{\partial T}{\partial t}$ will be considered. Finally, "two-dimensional analysis" will mean a discussion of the complete Eq. (16). In this note we will only consider cases of zero- and one-dimensional analysis. In the first three parts of this note the short expression "onedimensional analysis" will be used for "one-dimensional analysis of the first kind." That means we consider instead of Eq. (16) the special form

$G(\Delta T, I)=Q(\Delta T)-\frac{A}{P} \frac{d}{d x}\left[k(T) \frac{d \Delta T}{d x}\right]$

\subsubsection{Normalized Differential Equations for Ranges A, B, and C}

In order to demonstrate the essential points which we have in mind, it is sufficient to consider the simplest conditions of the one-dimensional analysis - namely linear cooling $(Q=h \Delta T)$ and constant heat conductivity (k). Under these conditions we can write Eq. (17) in the following normalized forms:

Range A:

$$
\begin{aligned}
& 0=\tau_{1}-\frac{1}{\beta^{2}} \frac{d^{2} \tau_{1}}{d x^{2}} ; \\
& \frac{1}{\beta^{2}} \frac{d^{2} \tau_{1}}{d x^{2}}=\tau_{1},
\end{aligned}
$$

$$
\beta^{2}=\frac{P h}{\Lambda k}
$$

Range B: $\quad g=\alpha i\left(i-1+\tau_{2}\right)=\tau_{2}-\frac{1}{\beta^{2}} \frac{d^{2} \tau_{2}}{d x^{2}}$,

$$
\frac{1}{\beta^{2}} \frac{d^{2} \tau_{2}}{d x^{2}}=(\alpha i-1)\left[\tau_{2}-\frac{\alpha i(1-i)}{\alpha i-1}\right]
$$


$\underline{\text { Range C }: ~} \quad \alpha i^{2}=\tau_{3}-\frac{1}{\beta^{2}} \frac{d^{2} \tau_{3}}{d x^{2}}$,

$$
\frac{1}{\beta^{2}} \frac{d^{2} \tau_{3}}{d x^{2}}=\tau_{3}-\alpha i^{2} .
$$

\subsection{3 $\tau(\mathrm{x})$ Characteristic for the Cold End Recovery Current}

We consider a superconducting composite wire which is infinitely long. A value of $\alpha>1$ and a value of $\beta$ are specified. The problem is to investigate whether an equilibrium state as shown in Fig. 5 is possible. The current (i) and the distance (a) are unknown.

Range A is characterized by:

$$
\begin{aligned}
& \lim \tau_{1}=0 . \\
& x_{1} \rightarrow-\infty \\
& \text { Because } i_{C u}=0, \\
& \tau_{1 \mid x_{1}}=0=\tau_{1,0}=1-i .
\end{aligned}
$$

Range C: For $\tau_{3}$ hold Eq. (25) and

$$
\left.\tau_{3}\right|_{x_{3}} \rightarrow+\infty=\alpha i^{2}
$$

Furthermore, we must take into account that the temperature $(T)$ of the wire must be a continuous function with a continuous differential quotient $\frac{d T}{d x}$ (otherwise an infinitely large axial heat flow would result). 
Therefore,

$\left.\frac{\mathrm{d} \tau_{1}}{\mathrm{dx}_{1}}\right|_{\mathrm{x}_{1}=0}=\left.\frac{\mathrm{d} \tau_{2}}{\mathrm{dx_{2 }}}\right|_{\mathrm{x}_{2}}=0$

and

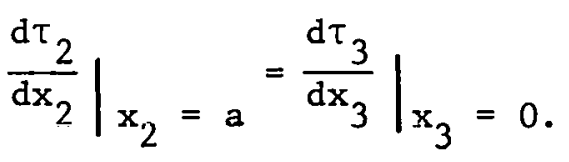

The solutions of the differential equations (18), (20), and (21) are:

$\tau_{1}=M_{1} e^{B x_{1}+N_{1 .}} e^{-\beta x_{1}}$,
$\tau_{2}=\frac{\alpha i(1-i)}{\alpha 1-1}+M_{2} e^{j \gamma x_{2}+N_{2}} e^{-j \gamma x_{2}}$,

with $j=\sqrt{-1}$ and $\gamma=\beta \sqrt{x i-1}$, and

$\tau_{3}=\alpha i^{2}+M_{3} e^{\beta x_{3}}+N_{3} e^{-\beta x_{3}}$

There are eight unknown quantities: $i, a, M_{1}, N_{1}, M_{2}, N_{2}, M_{3}$, and $\mathrm{N}_{3}$. The boundary conditions yield the following relations:

$$
\begin{aligned}
& M_{1}=1-i, \\
& N_{1}=0, \\
& 1-i=\frac{\alpha i(1-i)}{\alpha i-1}+M_{2}+N_{2}, \\
& B(1-i)=j \gamma\left(M_{2}-N_{2}\right), \\
& \frac{\alpha i(1-i)}{\alpha i-1}+M_{2} e^{j \gamma a}+N_{2} e^{-j \gamma a}=1.0,
\end{aligned}
$$


$B\left(\alpha i^{2}-1\right)=j \gamma\left(M_{2} e^{j \gamma a}-N_{2} e^{-j \gamma a}\right)$,

$\mathrm{M}_{3}=0$, and

$\alpha i^{2}+N_{3}=1.0$

We expect that it is possible to find the eight unknown quantities by means of Eqs. (33.1) to (33.8). If this is true, then there is only one equilibrium state possible for the situation represented in Fig. 5. The following numerical example shows that there are no special complications for solving this set of equations, despite the fact that the unknown are partly complex numbers.

In this case we call the resulting current (i) the "cold end recovery current." J.E.C. William ${ }^{9}$ uses the concept of the cold end recovery current for optimization of the winding of superconducting magnet coils. We do not know how he derived his basic equation.

The work of Maddock et al. concerning the cold end recovery current will be discussed in Sect. 3 .

\subsubsection{Numerical Example for the Temperature Profile $\tau(\mathrm{x})$ for Cold End Recovery}

For the following example we assume the values $\alpha=1.6$ and $\beta=2.7$ $\mathrm{cm}^{-1}$. Then, considering the boundary conditions Eqs. (33.1) to (33.8) we obtain:

$\gamma=1.614179 \mathrm{~cm}^{-1}$,

$\mathrm{a}=1.278624 \mathrm{~cm}$, and

$i=0.848386$.

$$
\begin{aligned}
& \text { Range A: } \tau_{1}=0.151614 e^{2.7 x_{1}}=(1-i) e^{\beta x_{1}} \text {. } \\
& \text { Range B: } \tau_{2}=0.481570\left[1.195686+\sin \gamma x_{2}-\sin \gamma\left(a-x_{2}\right)\right] \text {. } \\
& \text { Range } C: \quad \tau_{3}=1.151614-0.151614 \mathrm{e}^{-2.7 \mathrm{x}_{3}} \\
& =\alpha i^{2}+\left(1-\alpha i^{2}\right) e^{-\beta x_{3}} \text {. }
\end{aligned}
$$


$\left.\frac{\mathrm{d} \tau_{1}}{\mathrm{dx_{1 }}}\right|_{x_{1}=0}=\left.\frac{\mathrm{d} \tau_{2}}{\mathrm{dx}_{2}}\right|_{\mathrm{x}_{2}=0}=0.409358=\beta(1-i)$

and

$\left.\frac{\mathrm{d} \tau_{2}}{\mathrm{dx}_{2}}\right|_{\mathrm{x}_{2}=\mathrm{a}}=\left.\frac{\mathrm{d} \tau}{\mathrm{dx}}\right|_{\mathrm{x}_{3}=0}=0.409358=\beta\left(\alpha \mathrm{i}^{2}-1\right)$.

Equations (38), and (39) demonstrate the validity of the equation (see Sect. 3.3)

$\alpha i^{2}+i-2=0$

which shows that the value of $i$ does not depend on $\beta$. Equation (40) is the basic equation of J.E.C. Williams, ${ }^{9}$ which was later derived in a very elegant way by Maddock et al. ${ }^{3}$ That derivation will be discussed in detail in paragraph 3.3 of this note.

The shape of the temperature profile is symmetrical (see Fig. 6). We must keep in mind that this simple result holds only under the conditions of linear heat transfer and constant heat conductivity.

3. THE EQUAL AREA CONDITION BY MADDOCK ET AL.

3.1 THE DIFFERENTIAL EQUATION $y^{\prime}=F(y)$ AND CERTAIN KINDS OF STABILITY CRITERIA

As is well known, the method of separation of variables can be applied tor solving the differential equation

$y^{\prime}=F(y)$

when this equation is multiplied by

$\mathrm{s}=\frac{\mathrm{dy}}{\mathrm{dx}}$ 
Thus, $\quad s \frac{d s}{d x}=F(y) \frac{d y}{d x}$

and

$$
\frac{1}{2}\left(s^{2}-s_{0}^{2}\right)=\int_{y_{0}}^{y} F(y) d y .
$$

The second step is to proceed from

$$
\left(\frac{d y}{c x}\right)^{2}=s_{o}^{2}+2 \int_{y_{0}}^{y} F(y) d y
$$

to

$$
x-x_{0}=\int_{y_{0}}^{y} \frac{d y}{\sqrt{s_{0}^{2}+2 y_{y_{0}}} F(y) d y} .
$$

In the same way, one of the basic equations of classical mechanics can be derived. From

$m \frac{d^{2} x}{d t^{2}}=m \frac{d v}{d t}=F(x)$

follows

$\frac{m}{2}\left(v^{2}-v_{0}^{2}\right)=\int_{x_{0}}^{x_{0}} F(x) d x$,

which is the relation between kinetic energy and mechanical work. An explicit solution $[x(t)]$ has not been used.

For several other applications it is important that in those cases it is not necessary to know explictt solutions of all variables. There is a large group of modern mechanical stability theories (founded by the Russlall physlelsl Ljapunuv ${ }^{10}$ ) whlch make use of generailzed energy functions, and there is no necessity to know explicit solutions for all variables involved.

The same principle is being employed in many other fields of physics. As we shall see later the equal area condition of Maddock ct al. is an 
example of the endeavor to find conditions of stability without knowing explicit solutions.

3.2 DERIVATION OF THE EQUAL AREA CONDITION

Figure 1 of Ref. 3 considers forms of the $G(T, I)$ and $Q(T)$ characteristics and of the $T(x), G(x)$, and $Q(x)$ diagrams without restriction by the assumption of linear cooling. Similar characteristics and diagrams are shown here in Figs. $7(a)$ and $7(b)$. The reasoning of Maddock et al. is as follows. The starting point is Eq. (17) in the form

$\frac{A}{P} \frac{d}{d x}\left[k(T) \frac{d T}{d x}\right]=Q(T)-G(T, I)$

A term

$\mathrm{S}=k(\mathrm{~T}) \frac{\mathrm{dT}}{\mathrm{dx}}$

is introduced.

Multiplying Eq. (45) with $\mathrm{S}$ and integration yields

$$
\begin{aligned}
& \frac{A}{P} \int_{T}^{2} S d S=\frac{A}{2 F}\left(S_{2}^{2}-S_{1}^{2}\right)=\int_{T_{1}}^{T_{2}}[Q(T)-G(T, I)] k(T) d T, \\
& \text { with [see Fig. } 7(b)] \\
& T_{1}=11 \text { III } \\
& \quad x \rightarrow-\infty \\
& T_{2}=1 i m T \\
& \quad x \rightarrow+\infty
\end{aligned}
$$

If the assumption

$$
S_{1}=S\left(T_{1}\right)=S\left(T_{2}\right)=S_{2}=0
$$


is made, then

$\int_{T_{1}}^{T_{2}}[Q(T)-G(T, I)] k(T) d T=0$.

If $\mathrm{k}$ is assumed to be independent of temperature, then

$\int_{T_{1}}^{T_{2}}[Q(T)-G(T, I)] d T=0$.

Under the additional assumption [see Fig. 7(b)]

$Q\left(T_{1}\right)=G\left(T_{1}, I\right)$ and $Q\left(T_{2}\right)=G\left(T_{2}, I\right)$,

Eq. (51) corresponds to equal areas $a=b$ in Fig. 7(a). This is the equal area condition of Maddock et al. In normalized form Eq. (51) becomes

$\int_{\tau_{1}}^{\tau}[q(\tau)-g(\tau, i)] d \tau=0$.

At low temperatures, the heat conductivity $(\mathrm{k})$ is proportional to the absolute temperature $(T)$, i.e., $k=C T$. Therefore in Eq. (50) the factor $k(T) d T$ can be replaced by $\operatorname{CTdT}=\frac{C}{2} d\left(T^{2}\right)$. That means, if we plot $Q$ and $G$ not against $T$ but rather against $T^{2}$, we can still apply an equation corresponding to Eq. (51) and an equal area condition holds likewise. In contrast to the heat loss $Q(T)$, which is supposed to depend only on the temperature difference $\Delta T=T-T_{b}$, the Joule heat $G(T, I)$ depends on $T$ as a variable and on the current (I) as a parameter. It is important to keep in mind that the equal area condition is a condition for a critical transition value of the parameter I. Maddock et al. explain the meaning of the equal area stability condition in the following way: "If there are two equilibrium states, for example, one film boiling and the other nucleate boiling, then a change from one to the other can take place by the movement of the transition region between them along the conductors. The current for which this occurs is the cold end recovery current." 
In other words, Maddock et al. point.out that the transition occurs in the form of a traveling wave which has a certain front shape and which travels with a certain velocity. For these two. details, in Appendices 1 and 2 of Ref. 3, methods for estimating numerical values of the quantities involved are presented. The last paragraph of Appendix 1 reads: "Usually the order of magnitude $v$ is interesting and so the first approximation should be satisfactory. Furthermore, the other approximations (uniform temperature across the conductor, cooling rate independent of rate of change of temperature) probably invalidate any closer estimate of the velocity by this method."

The problem of the influence of "the uniform temperature distribution across the conductor" seems to be less difficult when modern, multifilament composite conductors are employed. Concerning the predicted difficulty because of the approximation "cooling rate independent of rate of change of temperature," Ref. 6 is of special interest. L. Dresner's work proves the intuitive assumption of Maddock et al. to be true.

\subsection{APPLICATION OF THE EQUAL AREA CONDITION TO THE CASE OF COLD END RECOVERY}

We assume linear heat transfer and constant heat conductivity. Then Eq. (53) can be represented by Fig. 8. The broken line shows the heat loss characteristic $\mathrm{q}(\tau)$ (straight line with a slope of $45^{\circ}$ ). Range A of the Joule heat generation characteristic $g(\tau)$ is represented by the horizontal portion $0 \leq \tau \leq \tau_{1,0}$ [corresponding to the interval $-\infty \leq x_{1} \leq 0$ of the $\tau(x)$ diagram in Fig. 5]. The sloping straight line section $\tau_{1,0} \leq \tau \leq 1$ represents Range $B$ (corresponding to $0 \leq x_{2} \leq a$ ), and the upper horizontal line section $\tau \geq 1.0$ (corresponding to $\left.0 \leq \mathrm{x}_{3}<+\infty\right)$ represents Range $\mathrm{C}\left(g=\alpha i^{2}\right.$ ) (compare Fig. 3 ). The $q(\tau)$ and the $g(\tau)$ diagrams intersect at point $U$ (unstable equilibrium). Its $\tau$ value $\tau^{*}$ follows from

$$
\begin{aligned}
& g=\alpha i\left(i-1+\tau^{*}\right)=\tau^{*} ; \\
& \tau *=\frac{\alpha i(1-i)}{\alpha i-1},
\end{aligned}
$$


In Fig. 8 the areas $a$ and $b$ are:

$a=\frac{1}{2} \tau_{1,0} \tau^{*}=\frac{1}{2}(1-i) \tau^{*} ;$
$b=\frac{1}{2}\left(\alpha i^{2}-1\right)\left(\alpha i^{2}-\tau^{*}\right)$.

From the equal area cundition $a=b$ follows

$\tau^{*}=\frac{\alpha i\left(\alpha i^{2}-1\right)}{\alpha i-1}$

If we consider the two expressions Eq. (54) and (56) for $\tau^{*}$, we obtain the cold end stability condition for the current (i)

$\alpha i^{2}+i-2=0 \quad$ or $\quad i=\frac{-1+\sqrt{1+8 \alpha}}{2 \alpha}=i_{r}$.

In Fig. 4 [zero-dimensional $v(i)$ characteristic], the value $i=\frac{1}{\sqrt{\alpha}}=$ $\frac{1}{\sqrt{1.5}} \doteq 0.816$ could mean an abrupt transition from Range $c$ to Range $A$ when the current ( $i$ ) is reduced. The cold end recovery current calculated by means of Eq. (57) is $i_{r}=0.869$, which means that due to the axial heat flow, the composite changes from the normal to the superconducting state when the current is only about $6.5 \%$ higher compared with $i=0.816$.

3.4 PERFORMANCE OF A RESISTANCE WIRE WITH COOLING BY NUCLEATE AND BY FILM BOILING LIQUID HELIUM

\subsection{1 $Q(\Delta \mathrm{T})$ and $\Delta \mathrm{T}(\mathrm{x})$ Diagrams}

In Ref. 3 it is pointed out that the transition from one zerodimensional equilibrium state to another can be easily demonstrated by considering a resistance wire [the resistance ( $R$ ) is supposed to change very little with $\Delta \mathrm{T}$ ] heated by a current (I) and cooled by liquid helium. With small currents and therefore small $\Delta \mathrm{T}$, cooling by nucleate boiling takes place. With higher currents, a change to cooling by film boiling can be expected. Therefore the special form of the cooling characteristic $Q(\Delta T)$ is, of course, important. 
In order to make our discussions as simple as possible we will consider the $Q(\Delta T)$ characteristic shown in Fig. 9. The regime of cooling by nucleate boiling is characterized by

$\mathrm{Q}_{\mathrm{n}}(\Delta \mathrm{T})=\mathrm{h}_{1} \Delta \mathrm{T}$ and $0 \leq \Delta \mathrm{T}<\Delta \mathrm{T}_{\mathrm{o}}$

We call $\Delta \mathrm{T}_{\mathrm{o}}$ the critical temperature difference. For the film boiling regime,

$\mathrm{Q}_{\mathrm{f}}(\Delta \mathrm{T})=\mathrm{h}_{2} \Delta \mathrm{T} \quad$ and $\quad \Delta \mathrm{T}>\Delta \mathrm{T}_{\mathrm{O}}$

The Joule heat produced per unit area is

$G(I)=\frac{R I^{2}}{P}$

For the nucleate boiling regime, $G\left(I_{n}\right)=Q_{n}$; for the film boiling regime, $G\left(I_{f}\right)=Q_{f}$.

For zero-dimensional equilibrium, $Q(\Delta T)=G(I)$; therefore we can expect (by first raising and then lowering the current) the cycle shown in Fig. 10. That means we notice one abrupt change $[(1) \rightarrow(2)]$ from nucleate to film boiling and another jump $[(4) \rightarrow(5)]$ back to nucleate boiling. The critical currents are $\mathrm{I}_{1,2}$ and $\mathrm{I}_{4,5}$. Considering the critical temperature difference $\Delta \mathrm{T}_{0}$ we obtain

$O_{1,2}=G\left(I_{1,2}\right)=n_{1} \Delta^{\prime} I_{u}^{\prime}$

and

$\mathrm{Q}_{4,5}-\mathrm{G}\left(\mathrm{I}_{4,5}\right)-\mathrm{h}_{2} \Delta \mathrm{T}_{\mathrm{o}}$

In Ref. 3 Maddock et al. point out that under consideration of a transition zone with axial heat flow and taking into account various boundary conditions, other transition cycles can be expected (shown in Fig. 11). In all these cases transitions $(6) \rightarrow(7)$ occur at a value $Q_{6,7}$ between $Q_{1,2}$ and $Q_{4,5}$ which can replace transition $(4) \rightarrow(5)$, transition $(1) \rightarrow(2)$, or both. For this transition instead of zerodimensional analysis, one-dimensional analysis must be applied. 
A long wire can be cooled on both ends [Fig. 11(a)]. The transitions $(1) \rightarrow(2)$ and $(6) \rightarrow(7)$ are expected. If the wire is cooled on one end and the other end is kept film boiling (by means of a small heater), transitions (6) $\vec{\ddagger}(7)$ without hysteresis should occur [Fig. 11(b)]. Finally, if both ends are heated the transitions $(7) \rightarrow(6)$ and $(4) \rightarrow(5)$ shown in Fig. 11(c) should take place. It is of interest that in Fig. 11 the condition $Q(\Delta T)=G(I)$ holds for the nucleate and the film boiling portions of the characteristic. For points on the transition lines from (6) to (7) and vice versa, $G(I)$ and $Q(\Delta T)$ are different because of the axial heat flow. This will be explained in detail in Sect. 4 of this note.

$\Delta \mathrm{T}(\mathrm{x})$ diagrams for transitions $(6) \rightarrow(7)$ and $(7) \rightarrow(6)$ are schematically shown in Fig. 12(a), 12(b), and 12(c). The horizontal lines $\Delta \mathrm{T}_{\mathrm{f}}$ represent one asymptote for the higher value of $\Delta \mathrm{T}, \Delta \mathrm{T}_{\mathrm{n}}$ the other asymptotic for the lower value. Figure 12 is only schematical, because the wire is supposed to be very long and the length of the transition zone is relatively very short.

3.4.2 Analytical Solution for the Transition Value of the Joule Heating Rate G (Based on the $Q(\Delta T)$ Diagram of Fig. 9)

For the zone of nucleate bolling $\left(\Delta \mathrm{T}_{1}<\Delta \mathrm{T}_{0}\right)$, the differential equation [see Eq. (17)] is

$G=\frac{\mathrm{RI}^{2}}{\mathrm{P}}=\mathrm{h}_{1} \Delta \mathrm{T}_{1}-\frac{\mathrm{Ak}}{\mathrm{P}} \frac{\mathrm{d}^{2} \Delta \mathrm{T}_{1}}{\mathrm{dx}_{1}^{2}}$

or

$$
\frac{1}{\delta^{2}} \frac{\mathrm{d}^{2} \Delta \mathrm{T}_{1}}{\mathrm{dx}_{1}^{2}}=\Delta \mathrm{T}_{1}-\frac{\mathrm{G}}{\mathrm{h}_{1}} ;
$$

$\beta_{1}=\sqrt{\frac{\mathrm{Ph}_{1}}{\mathrm{Ak}}}$. 
The solution is

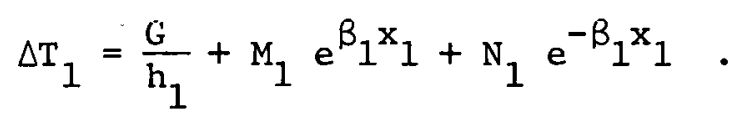

For $x_{1} \rightarrow-\infty$ we expect the asymptotic value $\frac{G}{h_{1}}$ and therefore $M_{1}=0$.

For $\mathrm{x}_{1}=0, \Delta \mathrm{T}_{1}$ is equal to the critical temperature difference $\Delta \mathrm{T}_{0}$. Therefore $\mathrm{N}_{1}=\Delta \mathrm{T}_{\mathrm{o}}-\frac{\mathrm{G}}{\mathrm{h}_{1}}$ and

$$
\Delta \mathrm{T}_{\perp}=\frac{G}{h_{1}}+\left(\Delta \mathrm{T}_{0}-\frac{G}{h_{1}}\right) e^{+\beta_{1} x_{1}} .
$$

In a similar way we obtain

$$
\begin{aligned}
& \Delta T_{2}=\frac{G}{h_{2}}-\left(\frac{G}{h_{2}}-\Delta T_{0}\right) e^{-B_{2} x_{2}} \text { and } \\
& B_{2}=\sqrt{\frac{P h_{2}}{A k}},
\end{aligned}
$$

with the asymptotic value $\frac{\mathrm{G}}{\mathrm{h}_{2}}$ (see Fig. 13). Because of the condition that the value of the axial heat flow must be a continuous function,

$\left.\frac{\mathrm{d} \Delta \mathrm{T}_{1}}{\mathrm{d \textrm {x } _ { 1 }}}\right|_{\mathrm{x}_{1}=0}=\left.\frac{\mathrm{d} \Delta \mathrm{T}_{2}}{\mathrm{dx_{2 }}}\right|_{\mathrm{x}_{2}=0}$

or

$\beta_{1}\left[\Delta T_{0}-\frac{G}{h_{1}}\right]-\beta_{2}\left[\frac{G}{h_{2}}-\Delta T_{0}\right]$.

The transition value of $G$ with the transition current $I^{*}$ is:

$G=G^{*}=G\left(I^{*}\right)=\frac{R\left(I^{*}\right)^{2}}{\Gamma^{\prime}}$

If we consider the values of $\beta_{1}$ and $\beta_{2}$ defined by Eqs. (62) and (65), respectively, from Eq. (67) follows

$G^{*}=\sqrt{h_{1} h_{2}} \Delta T_{0}$ 
This transition value $G^{*}$ corresponds to the $Q(\Delta T)$ transitions $(6) \stackrel{\rightarrow}{*}(7)$ of Fig. 11 .

\subsubsection{Check of the Equal Area Condition}

From Fig. 14 follow (for the areas $a$ and $b$ ):

$$
\begin{aligned}
& a=\frac{1}{2}\left(h_{1} T_{0}-G^{*}\right)\left(\Delta T_{o}-\Delta T_{n}\right) \quad \text { and } \\
& b=\frac{1}{2}\left(G^{*}-h_{2} T_{0}\right)\left(\Delta T_{f}-\Delta T_{o}\right)
\end{aligned}
$$

Considering the value of $\mathrm{G}^{*}[\mathrm{Eq} .(68)]$, we obtain

$$
\begin{aligned}
& \Delta \mathrm{T}_{\mathrm{n}}=\sqrt{\frac{\mathrm{h}_{2}}{\mathrm{~h}_{1}}} \Delta \mathrm{T}_{\mathrm{o}} \text { and } \\
& \Delta \mathrm{T}_{\mathrm{f}}=\sqrt{\frac{\mathrm{h}_{1}}{\mathrm{~h}_{2}}} \Delta \mathrm{T}_{\mathrm{o}} .
\end{aligned}
$$

Substituting these values in Eq. (69), we see immediately that

$$
\mathrm{a}=\mathrm{b}=\frac{1}{2}\left[\left(\sqrt{\mathrm{h}_{1}}-\sqrt{\mathrm{h}_{2}}\right) \Delta \mathrm{T}_{\mathrm{o}}\right]^{2} \text {. }
$$

This is the equal area condition applied to the case of a resistance wire when a transition takes place from nucleate to film boiling (or vice versa) of the liquid helium, which is used as coolant.

\section{DISCUSSION OF THE PRESUPPOSITIONS FOR THE VALIDITY OF THE EQUAL AREA CONDITION}

\subsection{THE LONG WIRE}

\subsubsection{Acceptable Temperature Tolerances. $\Delta T(x)$ vs $Q(\Delta T)$ Diagrams}

In the strict sense of the word, zero-dimensional solutions $G(\Delta T, I)=$ $Q(\Delta T)$ have no physical meaning. If we assume the simplest boundary condition for the two ends of the wire, namely $\Delta \mathrm{T}_{e}=\mathrm{T}_{e}-\mathrm{T}_{\mathrm{b}}=0$, the temperature distribution along a conductor of any finite length $2 \mathrm{a}$ leads to one-dimensional analysis. In order to decide whether or not the simplification to a zero-dimensional problem is acceptable, it is necessary 
to make some assumptions about the tolerable differences between the numerical values based on one-dimensional analysis and the correspondent values from the zero-dimensional solutions. We will illustrate this statement by the following simple example.

Through a piece of a nonsuperconducting wire of the length $2 \mathrm{a}$ with a circular cross section of diameter $D$ flows a current (I). The terminals are assumed to keep the two ends of the wire almost exactly at the bath temperature. The electrical resistance ( $R$ ) per centimeter length of the wire is supposed to be independent from not too large changes of T. Thus,

$G_{T}=\frac{R I^{2}}{P}=\frac{R I^{2}}{\pi D}$

if the entire circumference is cooled. With the boundary conditions $\Delta \mathrm{T}=$ 0 for $x=-a$ and for $x=+a$ we obtain [similar to Eq. (63)] the solution

$\Delta T=\frac{G}{h}\left(1-\frac{\cosh \beta x}{\cosh \beta a}\right)$ (see Fig. 15).

The highest temperature difference $\Delta \mathrm{T}_{M}$ occurs at the midplane:

$\Delta \mathrm{T}_{\mathrm{M}}=\left.\Delta \mathrm{T}\right|_{\mathrm{X}=0}=\frac{\mathrm{G}}{\mathrm{h}}\left(1-\frac{\mathrm{I}}{\cosh \beta \mathrm{a}}\right)$.

For $a \rightarrow \infty$ we obtain the value of the zero-dimensional analysis

$\Delta \mathrm{T}_{\mathrm{Mo}}=\frac{\mathrm{G}}{\mathrm{h}}$.

If we consider a certain percentage of the length of the wire, say $90 \%$, we can ask how much the value of $\Delta \mathrm{T}$ changes for $-0.9 \mathrm{a} \leq \mathrm{x} \leq+0.9 \mathrm{a}$.

Besides the values $\Delta \mathrm{T}_{\mathrm{M}}$ and $\Delta \mathrm{T}_{\mathrm{Mo}}$ we introduce the quantity

$\Delta \mathrm{T}_{\mathrm{m}}=\left.\Delta \mathrm{T}\right|_{\mathrm{x}=0.9 \mathrm{a}}=\frac{\mathrm{G}}{\mathrm{h}}\left(1-\frac{\cosh 0.9 B \mathrm{a}}{\cosh \beta \mathrm{a}}\right)$.

For a numerical example we assume:

$D=0.1 \mathrm{~cm}$,

$\mathrm{h}_{1}=0.9 \mathrm{Wcm}^{-2} \mathrm{~K}^{-1}$,

$\mathrm{k}^{1}=4 \mathrm{Wcm}^{-1} \mathrm{~K}^{-1}$, and therefore,

$B_{1}=3.0 \mathrm{~cm}^{-1}$. 
A value of about $\beta_{1}=3.0 \mathrm{~cm}^{-1}$ holds for nucleate boiling. For film boiling we assume $h_{2}=0.1 \mathrm{Wcm}^{-2} \mathrm{~K}^{-1}$ and $\beta_{2}=1.0 \mathrm{~cm}^{-1}$. Table 3 shows the ratios $\left(\Delta \mathrm{T}_{\mathrm{M}} / \Delta \mathrm{T}_{\mathrm{Mo}}\right)$ and $\left(\Delta \mathrm{T}_{\mathrm{m}} / \Delta \mathrm{T}_{\mathrm{Mo}}\right)$ for $\beta_{1}=3.0 \mathrm{~cm}^{-1}$ and for $\beta_{2}=1.0 \mathrm{~cm}^{-1}$.

For $B_{2}=1.0$ (film boiling) the values shown should be somewhat modified, because the two portions of the wire near the terminals are on low temperatures and therefore not in the film boiling but in the nucleate boiling state. However, the figures show the approximate differences when either nucleate or film boiling is considered.

We will tolerate a difference of around one percent between $\Delta \mathrm{T}_{\text {Mo }}$ and the lowest value of $\Delta \mathrm{T}$ (i.e., $\Delta \mathrm{T}_{\mathrm{m}}$ ) inside the zone of approximate homogeneity (here assumed to be $90 \%$ of the wire length). Table 3 shows that for nucleate boiling the wire with the specified data can be called "long" when its length (2a) is about $30 \mathrm{~cm}$. For film boiling a "long" wire has a length of about $100 \mathrm{~cm}$.

For a long wire, an excellent approximation for $\Delta \mathrm{T}_{\mathrm{m}}$ is

$\lim \Delta T_{m}=\frac{G}{h}\left(1-e^{\left.-\beta x_{m}\right)}\right.$,

$a \rightarrow \infty$

where $x_{m}$ is the distance between one end (terminal) of the wire and the end of the zone of approximate homogeneity, characterized by $\Delta T_{m}$ (see Fig. 16).

For instance with $\mathrm{a}=3 \mathrm{~cm}$ and $\beta_{1}=1.0$, Table 3 shows $\Delta \mathrm{T}_{\mathrm{m}} / \Delta \mathrm{T}_{\mathrm{Mo}_{0}}=$ 0.25768 . For $x_{m}=0.1$ and $a=0.3 \mathrm{~cm}$, Eq. (77) yields a value of 0.25918 . From $\beta^{2}=\frac{\mathrm{Ph}}{\mathrm{Ak}}$ follows

$\beta=2 \sqrt{\frac{h}{k}} \frac{1}{\sqrt{D}}$.

Using this equation, the numerical values shown in Table 3 for a $0.1-\mathrm{cm}-$ thick wire can be easily employed for wires with other D. For instance, the length of the zone of approximate homogeneity of a wire with circular cross section of $0.9-\mathrm{cm}$ diam is three times as long as following from Table 3 .

It must be kept in mind that the expression "long" should not be applied to the total length of the wire when equilibrium transitions determined by the equivalent area condition are considered. In Ref. 3 
the following remark is made: "A reasonable length of normal region is assumed so that each end may be treated independently." That means when the transition region moves along the wire, the smaller distance from the end to the transition region must be long; otherwise the presuppositions for the equal area condition are not satisfied. In other words, the transition process must be well under way before the equal area condition can be applied.

It is of interest to compare the $\Delta \mathrm{T}(\mathrm{x})$ characteristic (Fig. 15) with the $Q(\Delta T)$ characteristic of Fig. 17. In both diagrams, with increasing length $2 \mathrm{a}$ the value $\Delta \mathrm{T}_{\mathrm{M}}$ fast approaches the value $\Delta \mathrm{T}_{\mathrm{Mo}}$. However, the ratio of the length of the end zone $\left(0 \leq \Delta \mathrm{T} \leq \Delta \mathrm{T}_{\mathrm{m}}\right)$ to the length of the zone of approximate homogeneity $\left(\Delta \mathrm{T}_{\mathrm{m}} \leq \Delta \mathrm{T} \leq \Delta \mathrm{T}_{\mathrm{Mo}_{\mathrm{o}}}\right)$ converges fast to small values with increasing length $2 \mathrm{a}$, whereas likewise with increasing length $2 \mathrm{a}$ the temperature difference in the end zone $\Delta \mathrm{T}_{\mathrm{m}}$ fast approaches the maximum value $\Delta \mathrm{T}_{\mathrm{Mo}}$ when simultaneously the acceptable temperature difference $\left(\Delta \mathrm{T}_{\text {Mo }}-\Delta \mathrm{T}_{\mathrm{m}}\right)$ approaches zero (see Fig. 17).

\subsubsection{The Condition $\lim S=0$ : Integration Over Limits Located Inside the Wire Length}

Considering the definition of S [Eq. (46)], from Eq. (73) fol1nws

$\mathrm{S}=\mathrm{k} \frac{\mathrm{dT}}{\mathrm{d} \overline{\mathrm{x}}}=\mathrm{k} \frac{\mathrm{d} \Delta \mathrm{T}}{\mathrm{dx}}=-\mathrm{k} \cdot \hat{\mathrm{x}} \frac{\mathrm{G}}{\mathrm{h}} \frac{\sinh \beta \mathrm{x}}{\cosh \beta \mathrm{a}}$.

Therefore,

$\left.\mathrm{S}\right|_{\mathrm{x}}=0=0$ and

$\left.s\right|_{x=-a}=k \beta \frac{G}{h} \tanh \beta a=-\left.s\right|_{x=+a}$, and finally,

$\lim _{x \rightarrow-m} S=k \beta \frac{G}{h}=\lim (-S)$

The derivation of the equal area condition is based on the presupposition $\mathrm{S}_{1}=\mathrm{S}_{2}=0$. For a symmetrical arrangement, $\mathrm{S}_{2}=0$ follows from Eq. (80). 
However, Eq. (82) shows that the limits of the integration should not be the endpoints $(x= \pm a)$ of the wire but points located inside the wire with such a value $x=x_{s}$ that $S$ becomes sufficiently small [Eq. (79)]. Similar to Table 3 it would be possible to calculate $x_{s}$ values for various acceptable $S$ values. In addition, it must be kept in mind that similar to our previous remark concerning the location of points with acceptable $\Delta T$ differences, it is necessary that $x_{x}$ and $a-x_{s}$ are long enough, because on both sides of the transition region the conditions for an acceptable value of $S$ must be satisfied.

4.2 ONE-DIMENSIONAL EQUILIBRIUM STATES OF A LIQUID HELIUM-COOLED RESISTANCE WIRE CONSIDERING FINITE LENGTHS OF THE NUCLEATE AND OF THE FILM BOILING ZONES

\subsubsection{Basic Equations: The $G\left(\Delta \mathrm{T}_{\mathrm{M}}\right)$ Diagram}

In Sect. 3.4 of this note we discussed the equilibrium states of a resistance wire with both ends connected to well cooled terminals. We considered an infinitely long wire and used asymptotic solutions. It is possible to derive in a relatively simple way analytical solutions if we facilitate our task by assuming constant heat conductivity and if we employ the simple $Q(\Delta T)$ characteristic of paragraph 3.4.1. Of course, in order to check experimental results it is necessary to consider more realistic data for $k(T)$ and - as far as possible - for $Q(\Delta T)$.

We consider a symmetrical arrangement. The temperature profile $\Delta \mathrm{T}(\mathrm{x})$ is shown in Fig. 18. The length of the wire is $2 \ell$; the portion cooled by liquid helium in the nucleate boiling state is a; the portion $\mathrm{b}=\ell-\mathrm{a}$ is cooled by film boiling helium. For $0 \leq \mathrm{x}_{1} \leq \mathrm{a}$ we can use the following relations:

$\Delta T_{1}\left(x_{1}\right)=\frac{G}{h_{1}}+M_{1} e^{\beta_{1}} 1+N_{1} e^{-\beta_{1}} 1$.

The boundary conditions are

$\left.\Delta \mathrm{T}_{1}\right|_{\mathrm{x}_{1}=0}=0 \quad$ and 
$\left.\Delta \mathrm{T}_{1}\right|_{\mathrm{x}_{1}=\mathrm{a}}=\Delta \mathrm{T}_{\mathrm{o}}$

With $\phi_{1}=\beta_{1}$ a, we obtain the following value:

$\Delta \mathrm{T}_{1}=\frac{\mathrm{k}}{\mathrm{h}_{1}}\left[1-\frac{\cosh \phi_{1}\left(\frac{\mathrm{a}}{2}-\mathrm{x}\right)}{\cosh \frac{\phi_{1} \mathrm{a}}{2}}\right]+\frac{\sinh \phi_{1} \mathrm{x}}{\sinh \phi_{1} \mathrm{a}} \Delta \mathrm{T}_{0}$

For the first derivative of $\Delta \mathrm{T}_{1}$ we find

$s_{A}=\left.\frac{d \Delta T_{1}}{d x_{1}}\right|_{x_{1}=0}=\beta_{1}\left(\frac{\Delta T_{0}}{\sinh \phi_{1}}+\frac{G}{h_{1}} \tanh \frac{\phi_{1}}{2}\right)$

This is in accordance with Eq. (82), but it is in contrast to the asymptotic one-dimensional analysis where $s_{A} \rightarrow 0$ when $2 l \rightarrow \infty$. Furthermore, $s_{0}=\left.\frac{d \Delta T_{1}}{d x_{1}}\right|_{x_{1}=a}=\beta_{1}\left(\frac{\Delta T_{0}}{\tanh \pi_{1}}-\frac{G}{h_{1}} \tanh \frac{\pi_{1}}{2}\right)$.

In the zone of film boiling we measure the coordinate $x_{2}$ from the origin $\mathrm{O}_{2}$ (see Fig. 18). $\Delta \mathrm{T}_{2}$ is determined by

$\Delta T_{2}\left(x_{2}\right)=\frac{G}{l_{2}}+M_{2} e^{\beta_{2} x_{2}}+N_{2} e^{-\beta_{2} x_{2}}$.

The boundary conditinns are

$\left.\Delta \mathrm{T}_{2}\right|_{\mathrm{x}_{2}=0}=\Delta \mathrm{T}_{\dot{0}} \quad$ and

$\left.\frac{\mathrm{d} \Delta \mathrm{T}_{2}}{\mathrm{dx_{2 }}}\right|_{\mathrm{x}_{2}=\mathrm{b}}=0$

With $\phi_{2}=B_{2}$ b we obtain

$\Delta \mathrm{T}_{2}=\frac{\mathrm{G}}{\mathrm{h}_{2}}+\frac{\cosh \beta_{2}\left(\mathrm{~b}-\mathrm{x}_{2}\right)}{\cosh \beta_{2} \mathrm{~b}}\left(\Delta \mathrm{T}_{0}-\frac{\mathrm{G}}{\mathrm{h}_{\mathrm{o}}}\right)$

and 
$\left.\frac{\mathrm{d} \Delta \mathrm{T}_{2}}{\mathrm{dx_{2 }}}\right|_{\mathrm{x}_{2}=0}=\mathrm{s}_{\mathrm{o}}=\beta_{2}\left(\frac{\mathrm{G}}{\mathrm{h}_{2}}-\Delta \mathrm{T}_{\mathrm{o}}\right) \tanh \phi_{2}$.

The last equation considers the fact that the first derivative of $\Delta \mathrm{T}$ must be continuous. If we compare Eq. (87) with Eq. (91) we can calculate the value of $G$. The result is

$G=\sqrt{h_{1} h_{2}} \Delta T_{0} \frac{p \cosh \phi_{1}+\tanh \phi_{2}}{\tanh \frac{\phi_{1}}{2}+p \tanh \phi_{2}}$,

with

$\mathrm{p}=\frac{\beta_{1}}{\beta_{2}}=\sqrt{\frac{h_{1}}{h_{2}}}$.

From Eqs. (89) to (93) follows

$\Delta \mathrm{T}_{\mathrm{M}}=\left.\Delta \mathrm{T}_{2}\right|_{\mathrm{x}_{2}=\mathrm{b}}=\frac{1}{\cosh \phi_{2}}\left(\frac{\mathrm{G}}{\sqrt{\mathrm{h}_{1} \mathrm{~h}_{2}}} 2 \mathrm{p} \sinh ^{2} \frac{\phi_{2}}{2}+\Delta \mathrm{T}_{\mathrm{o}}\right)$,

$\Delta \mathrm{T}_{\mathrm{M}}=\frac{\Delta \mathrm{T}_{\mathrm{o}}}{\cosh \phi_{2}}+\frac{\mathrm{G}}{\mathrm{h}_{2}}\left(1-\frac{1}{\cosh \phi_{2}}\right)$.

For the zero-dimensional analysis we used diagrams showing $G=Q$ as functions of $\Delta \mathrm{T}$. In those cases $\Delta \mathrm{T}$ is independent of $\mathrm{x}$ [see $\mathrm{Fig}$. 2(a) and $3(a)$ ]. In the case of one-dimensional analysis (considering axial heat flow), $\Delta \mathrm{T}$ depends on $\mathrm{x}$. However, the $\Delta \mathrm{T}(\mathrm{x})$ characteristic is very flat when we approach the value $\Delta \mathrm{T}_{M}$ (zone of approximate homogeneity). Therefore, it makes sense in this case to employ $G\left(\Delta T_{M}\right)$ diagrams. This will be explained in detail in the following numerical examples.

\subsubsection{Numerical Examples of $G(a)$ and $G\left(\Delta T_{M}\right)$ Diagrams}

At the left side of Fig. 19, a $G(a)$ diagram is shown for a resistance wire with the data shown in Sect. 4.1 .1 and $a$ half-length $\ell=1 \mathrm{~cm}$. For nucleate boiling only, $a=1 \mathrm{~cm}$ and $b=0$, the portion of the $G(a)$ 
characteristic is a vertical straight line from $G=0$ to $G=0.7494$ [point ( $I$ ) in the figure]. When $G$ is raised over this value, an abrupt transition takes place to $a=0.1539$ [point (II)]; then follows a further decrease of the nuclear boiling and an increase of the film boiling zone (III). Now G can be decreased below (II) with increasing a and decreasing $b$ until point (VI) is reached. Here another transition occurs to point (VII), which indicates return to the state of nucleate boiling over the entire length of the wire.

In the $G(a)$ diagram, equilibrium states currespond to the vertical pare (O)-(1) of the diagram and to the part (IIII)-(VI). The part (VI)-(I) (broken line) is not stable; therefore, transition (VI)-(VII).

At the right side of Fig. 19 the zero-dimensional diagram $(0),(1),(2)$, $(3),(4),(5),(0)$ is shown. If we apply the equal area condition, the transition (4)-(5) is replaced by (6)-(7). However, in our case this is not realistic because of too short a wire length. If we plot the $G\left(\Delta T_{M}\right)$ diagram (see Fig. 19), we obtain curve (0), (I), (II), (III), (VI), (VII), (0), which shows the transition (VI)-(VII) - very different from $(6)-(7)$.

For $\ell=10 \mathrm{~cm}$ and for $\ell=100 \mathrm{~cm}$, similar diagrams are replesenled in Figs. 20 and 21. In both cases transitions (VI)-(VII) take place at $G=0.2250$. The transition $(6)-(7)$ occurs at exactly $G=0.225$, which means that the difference due to the finite lengths of the wires is practically zero.

In Figs. 20 and 21 the middle part of the $G(a)$ diagram is very flat, but Lliere is one minimum point approximately at the middle of $\&$ and the transition to nucleate boiling over the remaining length of the wire occurs abruptly at this point. This will become more clear if we consider the $\Delta \mathrm{T}(\mathrm{x})$ diagrams.

Figure 22 shows the $\Delta \mathrm{T}(\mathrm{x})$ diagram for $\ell=10 \mathrm{~cm}$. The curves were calculated by means of Eq. (83), (85), (86), (89), (91), and (92) using the $G$ values from Eq. (94). The temperature profiles are labeled as follows:

(I) $(\mathrm{a}=10 \mathrm{~cm})$ The entire wire is cooled by nucleate boiling with the maximum $G=0.675$ for this state. The solid line $(0)-(I)$ indicates a continuous rise of $G$ from zero to the maximum. 
(II) After the transition (I)-(II) only a small part of the length of the wire is cooled by nucleate boiling. The transition (I)-(II) is marked by a broken line. The end zone with nucleate boiling becomes continuously shorter when $G$ is raised to state (III) (solid line with arrow).

(VI) The value of $\mathrm{G}$ can be continuously lowered to any value above 0.22501 . In the figure the temperature profiles are shown for $G=0.26704$ and 0.22595 . The minimum value $G=0.22501$ corresponds to $\mathrm{a}=4.87 \mathrm{~cm}$. For $0 \leq \mathrm{x} \leqslant 4 \mathrm{~cm}, \Delta \mathrm{T}$ is almost exactly equal to $0.25 \mathrm{~K}$; for $4 \leqslant \mathrm{x} \leq$ $10 \mathrm{~cm}, \Delta \mathrm{T}$ increases to $\Delta \mathrm{T}_{\mathrm{M}} \stackrel{\dot{=}}{=} 2.2 \mathrm{~K}$.

(VII) The transition (VI)-(VII) is discontinuous (broken line). When $G$ becomes again larger than 0.22501 , the temperature of the right half of the wire changes abruptly to the temperature difference $\Delta \mathrm{T}=0.25 \mathrm{~K}$. The left half of the wire was already at $\Delta \mathrm{T}=0.25 \mathrm{~K}$.

Figure 23 shows the temperature profiles for a wire with $\ell=100 \mathrm{~cm}$. For equilibrium states for $G$ just a little larger than 0.225 , the $\Delta T(x)$ diagrams have geometrical shapes similar to a traveling wave. Theoretically this traveling wave can proceed only to about half of the wire because in the $G(a)$ diagram the part left of the minimum point does not correspond to stable equilibrium. Under this condition, with an extremely small increase of $G$, the entire right side of the traveling wave breaks down abruptly from $2.25 \mathrm{~K}$ to $0.25 \mathrm{~K}$. All these discussions were made from the point of view of strict one-dimensional analysis of the first kind, i.e., the term $\frac{A}{P} c(T)$ $\frac{\partial \Delta \mathrm{T}}{\partial \mathrm{t}}$ in Eq. (16) is disregarded. As soon as we consider that additional term in any appropriate way, we recognize that a traveling wave will proceed over the entire length of the wire.

5. ONE-DIMENSIONAL ANALYSIS OF THE SECOND KIND

\subsection{BASIC EQUATIONS}

\subsubsection{Computer Solutions vs Analytical Solutions}

The basic equation of the one-dimensional analysis of the second kind is the special form of Eq. (16):

$G(\Delta T, I)=Q(\Delta T)+\frac{A}{P} c(T) \frac{d T}{d t}$ 
Quantitative solutions which are supposed to be compared with experimental results can only be achieved by means of computer programs. However, to demonstrate qualitatively the essential points of the analysis it is sufficient to consider the solutions for the simplest cases, namely linear cooling and specific heat (c) being constant. In such cases analytical solutions can be derived.

\subsubsection{Example of a Resistance Wire Cooled by Liquid Helium (I Is a Linear Function of the Time)}

We will demonstrate the one-dimensional analysis of the second kind on the example of a resistance wire cooled with liquid helium. We assume that through the wire flows a current (I) which increases from $I=0$ at $t=0$ proportional to the time $(t)$. Thus,

$\frac{\mathrm{RI}^{2}}{\mathrm{Ph}}=n \mathrm{t}^{2}=\Delta \mathrm{T}+\lambda \frac{\mathrm{dT}}{\mathrm{dt}}$

and

$\eta=\frac{\mathrm{R}}{\mathrm{Ph}}\left(\frac{\mathrm{I}}{\mathrm{t}_{\mathrm{o}}}\right)^{2} ; \lambda=\frac{\mathrm{Ac}}{\mathrm{Ph}}$.

$I_{0}$ is the maximum current which can continuously flow before nucleate boiling changes to film boiling $\left(\Delta \mathrm{T}_{0}=0.75\right)$. With the initial condition $\Delta \mathrm{T}=0$ at $t=0$, the solution of Eq. (98) is

$\Delta T=\eta\left[t^{2}-2 \lambda t+2 \lambda^{2}\left(1-e^{\left.-\frac{t}{\lambda}\right)}\right]\right.$

We consider two numerical examples with data similar to those shown in Sect. 4.1.1.

$\begin{aligned} \mathrm{D}=0.1 \mathrm{~cm} ; & \mathrm{h}=0.9 \mathrm{Wcm}^{-2} \mathrm{~K}^{-1} ; \quad \Delta \mathrm{T}_{\mathrm{u}}=0.75 \mathrm{k} ; \\ \rho=1.5 \times 10^{-8} \mathrm{nhm} \mathrm{cm} ; & \kappa=1.25 \times 10^{3} \mathrm{Jcm}^{-3} \mathrm{~K}^{-1} ; \lambda=3.5 \times 10^{-5} \text { ser. (100) }\end{aligned}$

The maximum current with cooling by nucleate boiling liquid helium is

$\dot{\mathrm{I}}_{\mathrm{o}} \stackrel{\bullet}{=} 330 \mathrm{~A}$.

We assume that $I_{0}$ is reached at $t=t_{0}=10^{-3} \mathrm{sec}$. Then, 
$n t_{0}^{2}=\frac{R I_{o}^{2}}{P h}=\Delta T_{0} \quad$ and

$\eta=\frac{\Delta T_{0}}{t_{o}^{2}}=0.75 \times 10^{6} \mathrm{Ksec}^{-2}$

Equation (99) yields the following result: after one millisecond $\Delta \mathrm{T} \doteq 0.70 \mathrm{~K}$, i.e., about $7 \%$ below $\Delta \mathrm{T}_{\mathrm{o}}=0.75 \mathrm{~K}$. In order to reach the full value of $\Delta \mathrm{T}_{0}$ it is necessary to let the current increase during $1.036 \mathrm{msec}$ instead of $1.0 \mathrm{msec}$. These differences are very small.

We assume that $I_{0}$ is reached at $t=t_{0}=10^{-4}$ sec. Then $\eta=0.75 \mathrm{x}$ $10^{8} \mathrm{Ksec}^{-2}$. In this case after $0.1 \mathrm{msec} \Delta \mathrm{T} \doteq 0.40 \mathrm{~K}$ and it needs about $0.13 \mathrm{msec}$ in order to reach the value $\Delta \mathrm{T}_{0}=0.75$. Compared with the previous example, the differences are appreciably larger.

\subsubsection{Equations for Superconducting Composites (Ranges A, B, and C)}

When we consider superconducting composites we add another simplification. Instead of working with continuous time functions [ $I(t)]$ as in the previous examples, we will assume that at $t=0$ the current (I) changes very fast from one to another constant value. We will express this assumption by saying, "I $(-0)$ changes to $I(+0) . "$

In normalized form the ranges $A, B$, and $C$ are characterized by the following differential equations.

Range A: $\quad 0=\tau_{1}+\lambda \frac{d \tau_{1}}{d t}$

$$
\begin{aligned}
& \text { or } \frac{\mathrm{d} \tau_{1}}{\mathrm{dt}}=-\frac{1}{\lambda} \tau_{1} \text {; } \\
& \lambda=\frac{\mathrm{Ac}}{\mathrm{Ph}} \text {. }
\end{aligned}
$$

Range B: $\quad g=\alpha i\left(i-1+\tau_{2}\right)=\tau_{2}+\lambda \frac{d \tau_{2}}{d t}$

$$
\text { or } \frac{\mathrm{d} \tau_{2}}{\mathrm{dt}}=\frac{\alpha i-1}{\lambda}\left[\tau_{2}+\frac{\alpha i(i-1)}{\alpha i-1}\right] \text {. }
$$


Range $\mathrm{C}: \quad \alpha i^{2}=\tau_{3}+\lambda \frac{\mathrm{d} \tau_{3}}{\mathrm{dt}}$

$$
\text { or } \frac{d \tau_{3}}{d t}=-\frac{1}{\lambda}\left(\tau_{3}-\alpha i^{2}\right) \text {. }
$$

In these equations $i$ means the constant current after the fast change, namely $i(+0)$.

These differential equations have the form

$\frac{d y}{d x}=A(y+B)$

The solution is

$y=C e^{A x}-B$

with the integration constant $\mathrm{C}$.

5.2 ONE-DIMENSIONAL ANALYSIS OF THE SECOND KIND APPLIED TO A COMPOSITE WITH $\alpha<1.0$

We consider the following two examples with $\alpha=0.5$ (compare Fig. 4).

(1) We start with the equilibrium state $i(-0)=1.0$ and change very fast to the constant value $i(+0)=i_{0}=1.1$ (which is smaller than $1 / \sqrt{0.5}=\sqrt{2}$ ). Since we stay in Range B, from Eq. (103) follows with $\left.T_{2}\right|_{t_{2}}=u=0$,

$\tau_{2}=0.1 \dot{2}\left(1-e^{\frac{0.45}{\lambda}} t_{2}\right)$.

For zero-dimensional thermal equilibrium the value of $\tau_{2}$ with $i_{0}=1.1$ is

$\tau_{2}=\frac{\alpha i(i-1)}{1-\alpha i}=0.12$.

This means that with a very fast change of $i=1.0$ to $i_{0}=1.1$, the variable changes asymptotically from zero to the zero-dimensional value of thermal equilibrium. 
(2) We start again with $i=1.0$ and change very fast to $i_{0}=1.8$ (which is larger than $\sqrt{2}$ ). We expect to pass through Range $B$ to Range $C$. For Range $B$ we obtain

$\frac{\mathrm{d} \tau_{2}}{\mathrm{dt}}=-\frac{0.1}{\lambda}\left(\tau_{2}-7.2\right)$

With $\left.\tau_{2}\right|_{t_{2}}=0=0$ the solution is

$\tau_{2}=7.2\left(1-e^{-\frac{0.1}{\lambda} t_{2}}\right)$.

Range $C\left(\tau_{3}=1\right)$ is reached at the time

$t_{2}=1.495317 \lambda$

For Range C [Eq. (104)]

$\frac{\mathrm{d} \tau_{3}}{\mathrm{dt}}=-\frac{1}{\lambda}\left(\tau_{3}-1.62\right)$

must be satisfied. We start to count the time $\left(t_{3}\right)$ from the moment when

$\tau_{2}=\tau_{3}=1.0$ is reached. Therefore, the initial condition for Eq. (109)

is $\left.\tau_{3}\right|_{t_{3}}=0=1.0$ and the solution of $\mathrm{Eq}$. (109) is

$\tau_{3}=1.62-0.62 \mathrm{e}^{-\frac{t_{3}}{\lambda}}$.

The value 1.62 with $\alpha i_{0}^{2}$ will be reached asymptotically.

5.3 ONE-DIMENSIONAL ANALYSIS OF THE SECOND KIND APPLIED TO A COMPOSITE WITH $\alpha>1.0$

In Sect. 2.1.2 we applied zero-dimensional analysis to the performance of a superconducting composite. We assumed current regulation and for $\alpha>1.0$ we mentioned a jump of the voltage (v) when $i$ changes from just below 1.0 to just above that value. The unstability criterion $\frac{\mathrm{dv}}{\mathrm{di}}<$ 0 seems to be valid; however, it is desirable to discuss this matter also from another point of view here. 
We consider the following example with $\alpha=1.5$. We start with $i(-0)=$ 1.0 and raise the current very fast to the value $i(+0)=1.05$. Since the value $\tau(-0)=\tau(+0)=0$, from Eq. (6) follows $v(+0)=i-1=0.05$. For Range B [Eq. (103)] we obtain the following numerical values.

$\frac{\mathrm{d} \tau_{2}}{\mathrm{dt}}=\frac{0.575}{\lambda}\left(\tau_{2}+0.136957\right)$

With $\left.\tau_{2}\right|_{t_{2}}=0=0$,

$\tau_{2}=0.136957\left(\mathrm{e}^{\frac{0.575}{\lambda} t_{2}}-1\right)$.

The value $\tau_{2}=1$ is reached at the time

$t_{2}=3.680777 \lambda$

The value $i_{0}=1.05$ corresponds to $\tau_{2}=\alpha i_{o}^{2}=1.5 \times 1.05^{2}-1.65375$.

Therefore, operation in Range $C$ must be taken into account. From

Eq. (104) follows

$\frac{\mathrm{d} \tau_{3}}{\mathrm{dt}}=-\frac{1}{\lambda}\left(\tau_{3}-1.65375\right)$,

with the boundary condition $\left.\tau_{3}\right|_{t_{3}}=0=1.0$. Therefore,

$\tau_{3}=1.65375 \quad 0.65375 \mathrm{c}^{-\frac{\tau_{3}}{\lambda}}$.

The value $\alpha i_{0}^{2}$ will be reached asymptotically.

If we repeat the calculation for $t_{2}$ with $i_{0}=1.01$, we obtain the value

$t_{2}^{\prime}=6.903222 \lambda$

We notice that with a smaller value of $|i(+0)-i(-0)|$ the value of $t_{2}$ increases slower.

Another interesting case is the performance of a composite with $\alpha>1.0$ when in Range $C$ the current is reduced to a value $i_{0}<1 / \sqrt{\alpha}$. 
When linear cooling is assumed, Range $C$ is characterized by $\tau=\alpha_{i}^{2} \geq 1.0$. From $i_{0}<1 / \sqrt{\alpha}$ follows $\tau<1.0$ and we cannot expect to be in the Range $C$. For $\alpha=1.5$ we found $i=1 / \sqrt{\alpha} \doteq 0.816$. When we reduce $i$ to a somewhat lower value, for instance to $i_{0}=0.8$, Eq. (12), which characterizes Range $B$, yields

$\tau=\frac{\alpha i_{0}\left(i_{0}-1\right)}{1-\alpha i_{0}}=1.2$.

Therefore, Range $B$, i.e., $0 \leq \tau \leq 1.0$, is also not possible. Finally, we could assume that when $\tau$ becomes a little smaller than 1.0, a fast jump from Range $C$ to Range A will occur. With a fast jump the temperature $\tau=1.0$ would not change considerably and the superconducting current and herewith the total current (i) should be almost equal to zero since $\tau=1.0$. This contradicts the assumptions of current regulation because we assumed that the current has been regulated from $i=1.0$ to $i=0.8$. We see that in this case zero-dimensional analysis does not work. A way out of this dilemma is the cold end recovery.

Calculations with continuously changing $i$ are, of course, more realistic than calculations with the current jumps $i(-0) \rightarrow i(+0)$. For the simplest cases (i.e., the resistance wire cooled by liquid helium when the current is linearly increased), analytical solutions are possible. In order to predict the performance of compound conductors with continuously variable currents, numerical computation by means of computer programs cannot be avoided. However, the simple analytical solutions under the assumptions of current jumps shown here yield some valuable information. It might be of interest to replace continuous functions $[i(t)]$ by a sequence of little jumps and to base computer programs on equations similar to those presented in this section. of course, an important problem is to find out in which cases one-dimensional analysis of the second kind should be taken into account instead of considering only a traveling wave solution. 


\section{IS THE EQUAL AREA CONDITION A SUFFICIENT CRITERION FOR CRYOSTATIC STABILIZATION?}

In Ref. 8 the definition of cryostatic stabilization starts with the following sentence: "Stabilization achieved by cladding a superconductor with a large enough amount of metal of high electrical conductivity and providing sufficient cooling that any part of it which becomes normal reverts to the resistanceless state without current having to be reduced."

For a moment we will concentrate our attention to the words "any part of it becomes normal." There is no special reason mentioned why a part of a superconducting composite becomes normal, but currents which are too high and external heat influxes can be the causes. All of these things (heat production by slipping of turns in the winding, use of heaters within the winding for stability tests, etc.) are so well known that an elaboration on these points here would be a waste of time. I would like only to refer for an example to the classical investigations of Stekly et al. in Ref. 2 .

The equal area condition does not deal with external heat sources and does not consider any kind of time functions. Therefore, it is clear that the equal area condition alone cannot be a sufficient criterion for cryostatic stability. This statement is so straightforward that the question arises whether very careful discussions of many details connected with the equal area condition make sense at all. I would like to answer this question with yes. The problem of cryostatic stability is so complex and so many points must still be cleared up that a careful discussion of many details which are without doubt parts of the problem of cryostatic stability should be carefully considered.

\section{SUMMARY}

- A special form of zero-dimensional analysis is to find equilibrium states of very thin and very long superconducting composite wires by intersecting their $q(\tau)$ and $g(\tau, i)$ characteristics. This method can be applied to experimental $q(\tau)$ characteristics of any shape. Furthermore, it has the advantage of familiarizing with the way of reasoning which leads to the derivation of the equal area condition. 
For educational purposes this type of zero-dimensional analysis is demonstrated in great detail in this note. The examples show that this method is rather cumbersome and should be replaced by calculations [Eqs. (13) and (14)] if the $q(\tau)$ relation can be expressed analytically - for instance, by terms $M_{k} e^{m_{k} \Delta T}$ for successive $\Delta \mathrm{T}$ intervals (see Fig. 6 of Ref. 6).

- By means of asymptotic one-dimensional analysis the complete temperature profile $[\Delta \mathrm{T}(\mathrm{x})]$ (superconducting, current sharing, and normal zones) for the case of cold end recovery of a superconducting composite under the assumption of linear cooling can be determined analytically. The necessary set of equations is derived and a numerical example is shown in detail. Even in this simple case relatively much computer work has to be done.

- Well known stability criteria of theoretical mechanics and other parts of physics are mentioned which do not need explicit solutions for all variables involved. The equal area condition by Maddock et al. belongs to this group. A derivation based on Ref. 3 is given in this note. The equal area condition can be used to find the cold end recovery and the breakaway currents (Ref. 4) of a superconducting composite in a simple way. The equal area condition is of special advantage when the $q(\tau)$ characteristic has an uncommon shape because of special surface conditions of the wire (see Ref. 4). However, the equal area condition does not yield information about the temperature profile and the time dependence - for instance, the velocity of propagation. This information cannot be expected because the variables $x$ and $t$ are not used in the equal area condition.

- In order to demonstrate as simply as possible various points of interest, a special form of the $Q(\Delta T)$ characteristic (compare Ref. 7) is used in this note. Thus, analytical expressions for the complete asymptotic temperature profile of a very thin and very long resistance wire can be derived. The cooling is by liquid helium partly with nucleate, partly with film boiling The validity of the equal area condition is proved for this special case. 
- Several general presuppositions for the validity of the equal area condition are discussed. The exact meaning of a long wire is demonstrated, and it is shown that the asymptotic solutions (on which the equal area condition is based) need a revision of the formulation of their boundary conditions (for mathematical cleanliness).

- Using the $Q(\Delta T)$ characteristic mentioned above, analytical solutions for the performance of a liquid helium-cooled resistance wire of finite length can be derived. Considering the zones of variable length "a" for cooling by nucleate boiling and length "b" by film boiling, a complete cycle is described and compared with the cycle $(0),(1),(2),(3),(4),(5),(0)$ of zero-dimensional analysis of an infinitely long wire. The characteristic points for the wire of finite length found by one-dimensional analysis are: $(0),(\mathrm{I})$ nucleate boiling only; transition (I), (II) to mainly film boiling; branch (III) for increasing and decreasing current. Instead of transition (4), (5) of the infinitely long wire there is a transition (VI), (VII) with higher $\Delta \mathrm{T}$. (VII), (0) is with nucleate boiling only. $G(a), G\left(\Delta T_{M}\right)$, and complete temperature profiles $\Delta T(x)$ for wires of three different lengths are shown. The (VI), ( $\mathrm{VIL}$ ) transition of even medium long wires approaches very closely the performance expected by the equal area condition.

- The equal area condition holds for the transitions $(6) \vec{t}(7)$ from one to another asymptotic zero-dimensional equilibrium state. Since only one independent variable $(\Delta \mathrm{H})$, one parameter $(\mathcal{H})$, and two dependent variables $[Q(\Delta T)$ and $G(\Delta T, I)]$ are concerned, no statements about $\Delta \mathrm{T}(\mathrm{x}, \mathrm{t})$ can be made. Special solutions for the transitions $(6) \vec{t}(7)$ are traveling wave solutions which cannot be determined by the equal area condition alone (see Ref. 6). Tilme dependence can be expected in different forms. Even if we could be satisfied with zero-dimensional solutions and if we could predict correctly traneitione between thom by moane of traveling wavco, there are additional points which can be understood only by considering the dependence of time. Since a comprehensive discussion of all the practically interesting solutions of the partial 
differential equation [Eq. (16) of this note] is a very difficult task, we restricted ourselves to consider one-dimensional analysis of the second kind. That means to find for a thin and very long wire solutions $\Delta T(t)$. One analytical solution (with linear cooling) for a resistance wire when the current (I) is a linear function of the time $(t)$ is presented and discussed in detail in this note. For very thin and very long superconducting composites, analytical solutions can be found easily when the current is a step function in respect to time. That means one constant value $[I(-0)]$ changes very fast to another constant value $[I(+0)]$. Examples are shown in this note. Of special interest is the jump of $v(i)$ for $\alpha>1.0$ at $i=1.0$. It might be possible to obtain in a relatively simple way computer codes for variable I by employing our solutions for series of successive little current jumps.

- As is well known the cryostatic stability of superconducting composites depends on the time functions of the external heat influx. The equal area condition does not deal with external heat influx and does not consider any kind of time functions. Therefore, the equal area condition is not a sufficient criterion for cryostatic stability. Nevertheless, we believe that the careful discussion of many details presented here can contribute towards a better understanding of the problem of cryostatic stability.

\section{ACKNOWLEDGEMENT}

I would like to express my special thanks to $\mathrm{Mr}$. Helmut Haslacher for his most valuable assistance in preparing this report. 
Table 1. Evaluation of $g(\tau, i)$ diagrams shown in Fig. 2 for finding equilibrium states (zero-dimensional with linear cooling)

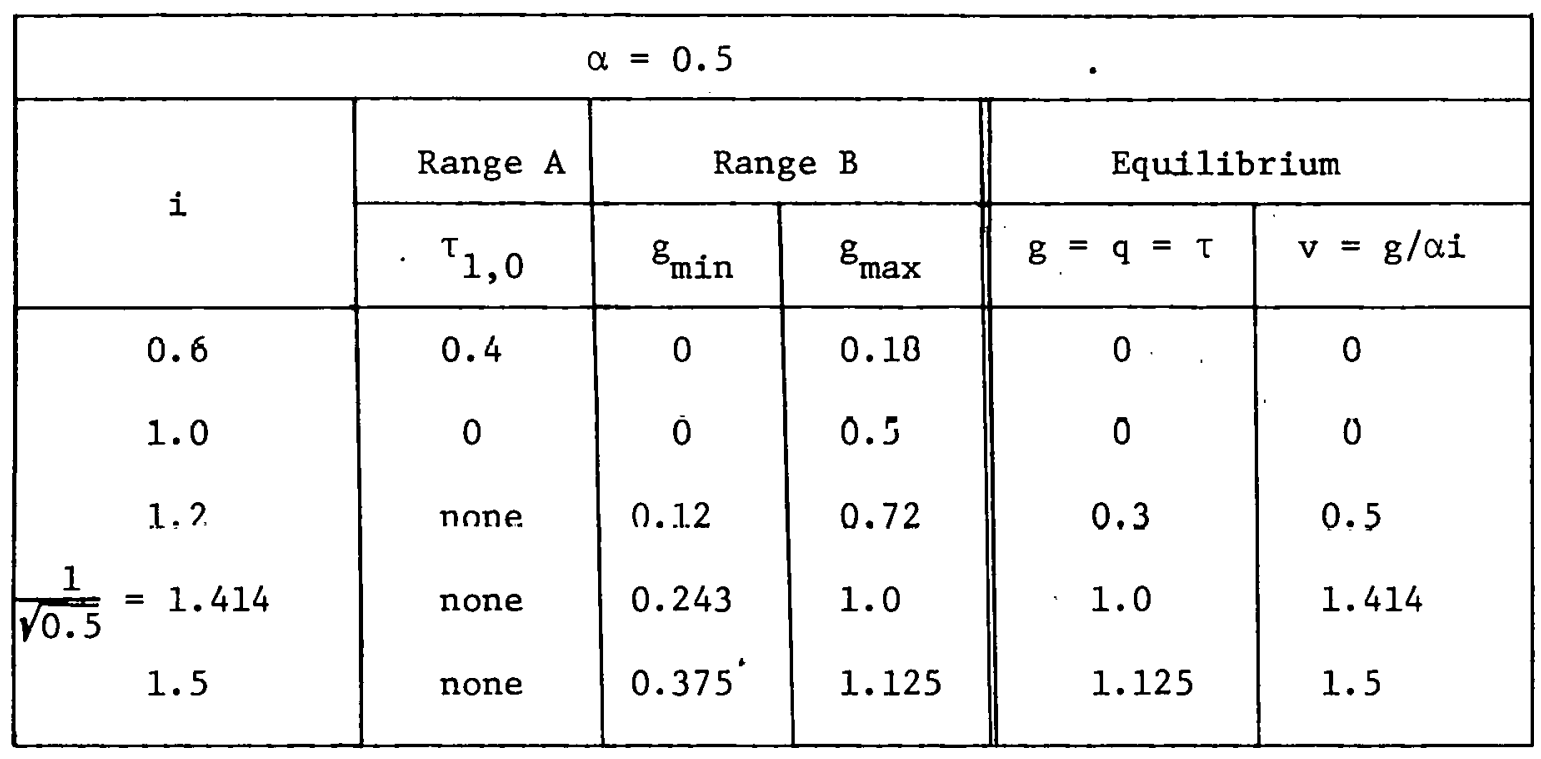

Table 2. Evaluation of $g(\tau, i)$ diagrams shown in Fig.: 3 for finding equilibrium states (zero-dimensional with linear couling)

\begin{tabular}{|c|c|c|c|c|c|c|}
\hline \multicolumn{7}{|c|}{$\alpha=0.5$} \\
\hline \multirow{2}{*}{\multicolumn{2}{|c|}{1}} & \multirow{2}{*}{$\frac{\text { Range } A}{\tau_{1,0}}$} & \multicolumn{2}{|c|}{ Range $B$} & \multicolumn{2}{|c|}{ Equilibibrium } \\
\hline & & & $\mathrm{g}_{\mathrm{min}}$ & $\mathrm{g}_{\max }$ & $g=q-\tau$ & $v=g / \alpha i$ \\
\hline & 0.6 & 0.4 & 0 & 0.54 & 0 & 0 \\
\hline$\frac{1}{\sqrt{1.5}}=$ & $=0.816$ & 0.184 & 0 & 1.0 & $0,1.0$ & $0,0.816$ \\
\hline & 0.9 & 0.1 & 0 & 1.215 & $0,(0.386), 1.215$ & $0,(0.286), 0.9$ \\
\hline & 1.0 & 0 & 0 & 1.5 & $0,1.5$ & $0,1.0$ \\
\hline & 1.1 & none & 0.165 & 1.81 .5 & $1 . .81 .5$ & 1.1 \\
\hline
\end{tabular}


Table 3. Ratios of maximum temperature differences $\Delta T_{M}$ and of the minimum temperature differences $\Delta \mathrm{T}_{\mathrm{m}}$ (determined by one-dimensional analysis) to the temperature differences $\Delta \mathrm{T}_{\text {Mo }}$ (found by zero-dimensional analysis)

\begin{tabular}{|c|c|c|c|c|}
\hline & \multicolumn{2}{|c|}{$\beta=3,0$} & \multicolumn{2}{c|}{$\beta=1,0$} \\
\hline $\mathrm{a}(\mathrm{cm})$ & $\Delta_{\mathrm{M}} / \Delta \mathrm{T}_{\mathrm{Mo}}$ & $\Delta_{\mathrm{m}} / \Delta \mathrm{T}_{\mathrm{Mo}}$ & $\Delta \mathrm{T}_{\mathrm{M}} / \Delta \mathrm{T}_{\mathrm{Mo}}$ & $\Delta_{\mathrm{m}} / \Delta \mathrm{T}_{\mathrm{Mo}}$ \\
\hline \hline 1 & 0,90067 & 0,25768 & 0,35195 & 0,07128 \\
3 & 0,99975 & 0,59343 & 0,90067 & 0,25768 \\
5 & 1,00000 & 0,77687 & 0,98652 & 0,39342 \\
10 & 1,00000 & 0,95021 & 0,99991 & 0,63212 \\
15 & 1,00000 & 0,98889 & 1,00000 & 0,77687 \\
20 & 1,00000 & 0,99752 & 1,00000 & 0,86466 \\
40 & 1,00000 & 0,99999 & 1,00000 & 0,98168 \\
50 & 1,00000 & 1,00000 & 1,00000 & 0,99326 \\
\hline
\end{tabular}


ORNL/DWG/FED-77756

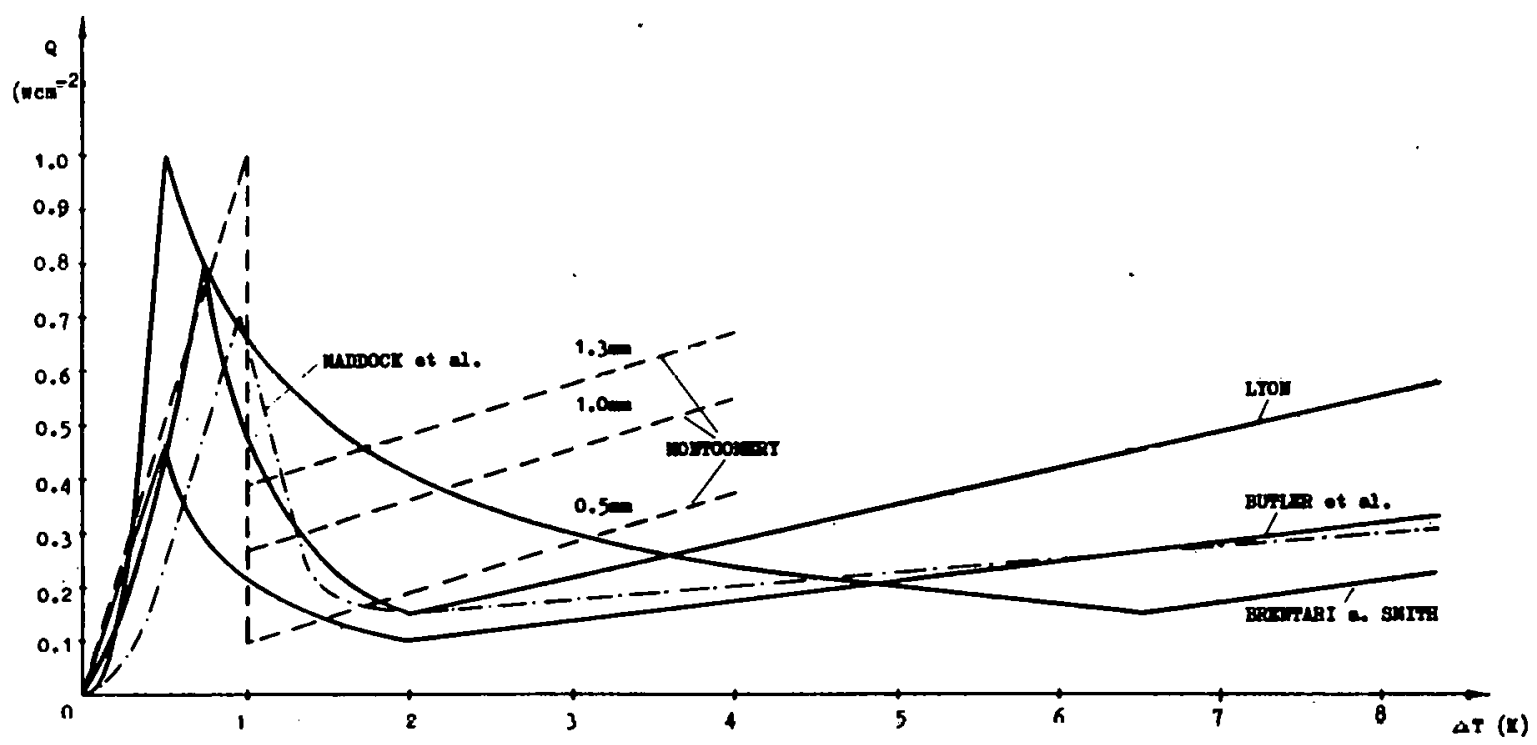

Fig. 1. $Q(\Delta T)$ characteristics from Refs. 3, 4, 6, and 7 (drawn in lincar scale). 
ORNL/DWG/FED-77746

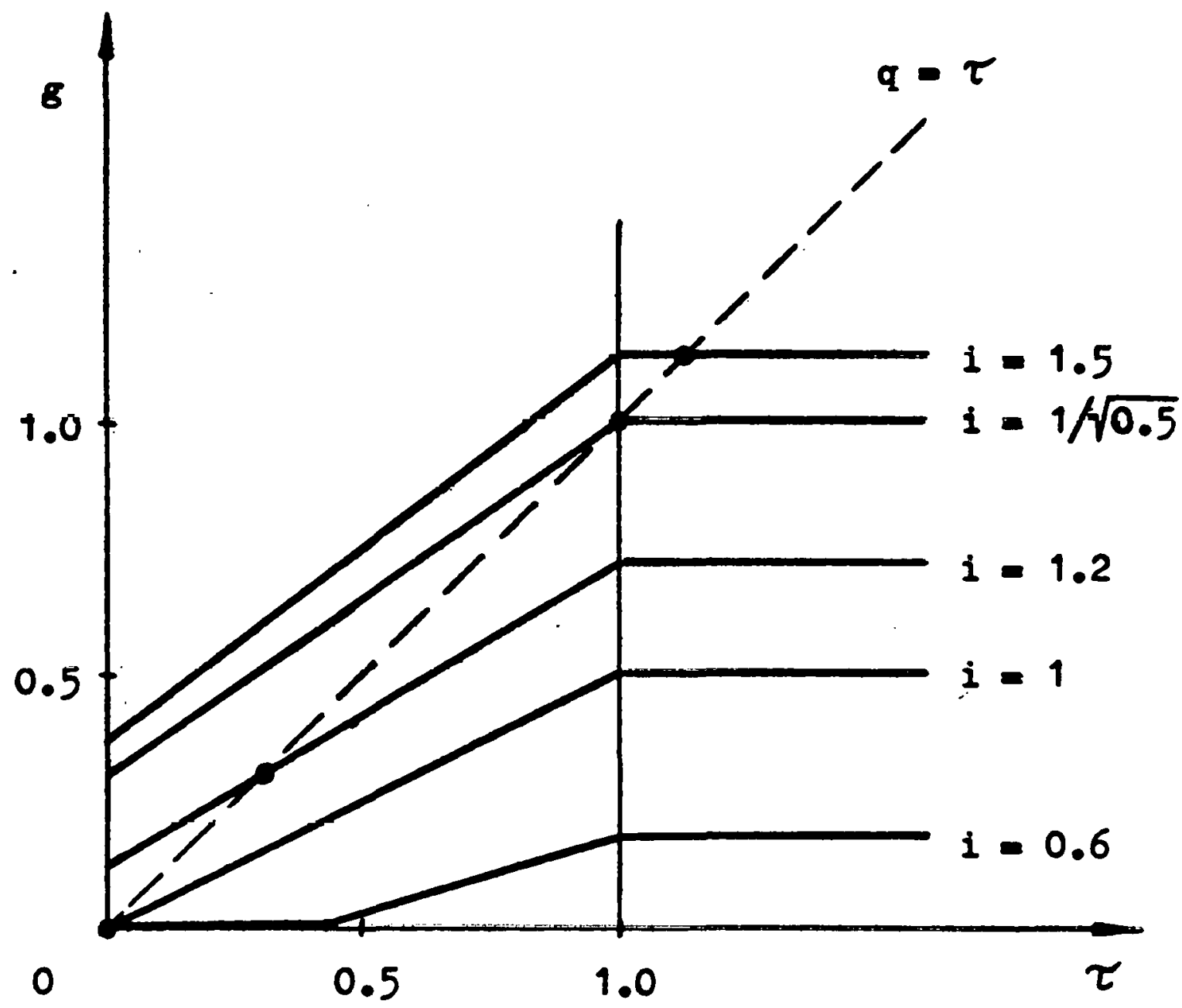

Fig. 2. $g(\tau)$ diagrams for $\alpha=0.5$ with various values of the parameter $i$ and $q(\tau)$ diagrams for linear cooling (zerodimensional analysis). 
ORNL/DWG/FED-77735

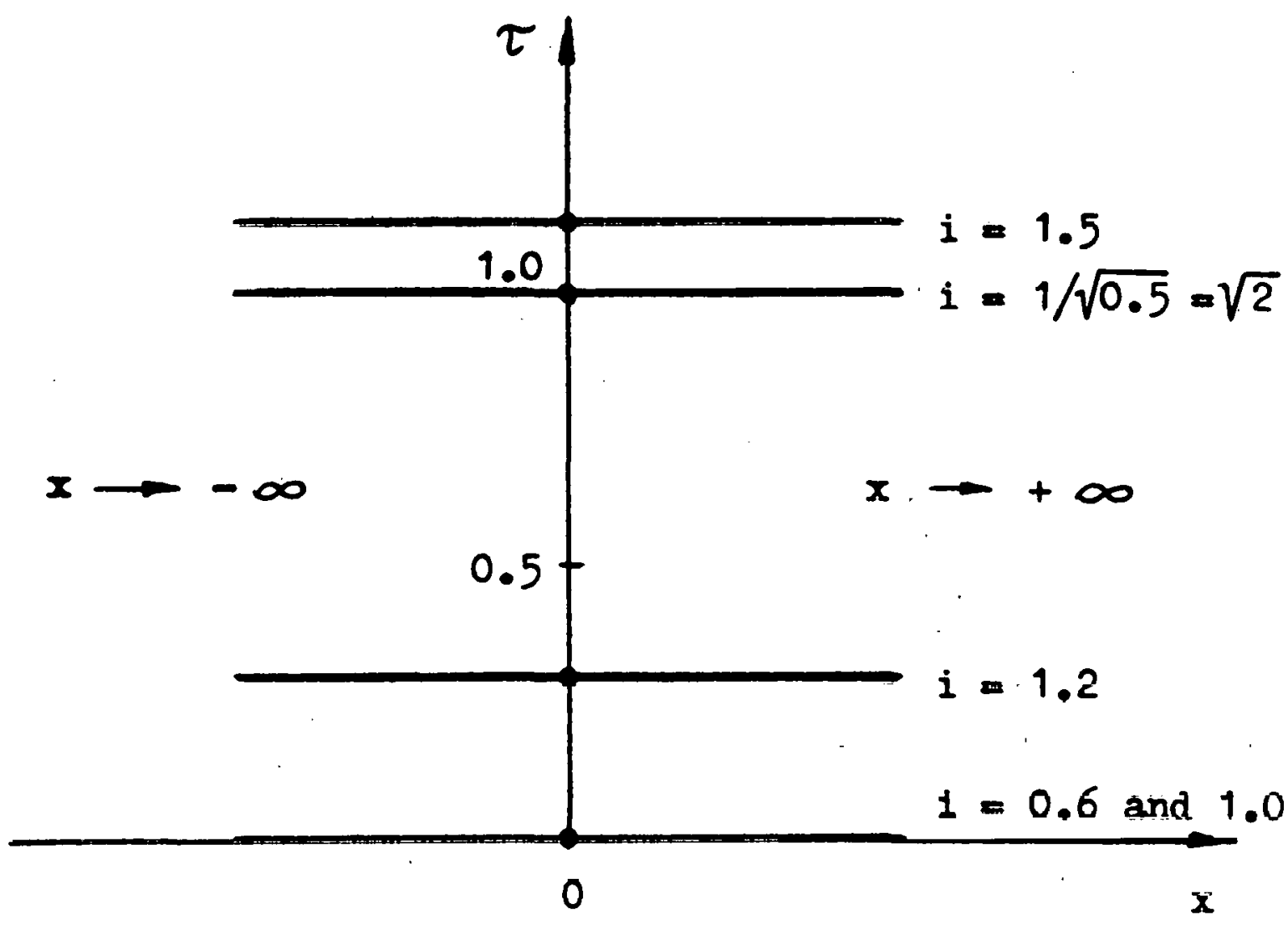

Fig. $2(a) . \quad \tau(x)$ diagrams of stable equilibrium states for $\alpha=0.5$ (zero-dimensional analysis). 
ORNL/DWG/FED-77737

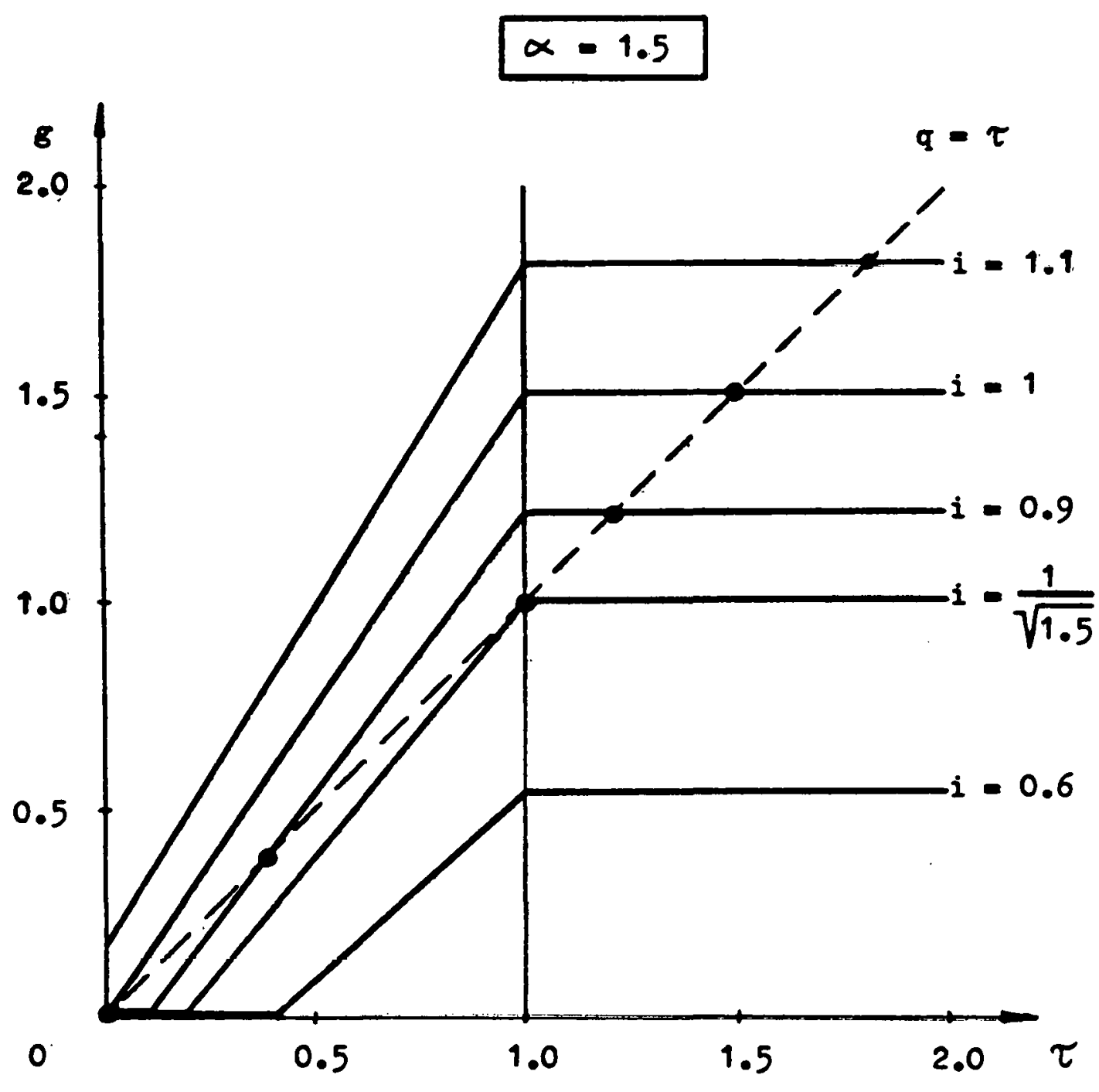

Fig. 3. $g(\tau)$ diagrams for $\alpha=1.5$ with various values of the parameter $i$ and $q(\tau)$ diagrams for linear cooling (zerodimensional analysis). 


\section{ORNL/DWG/FED-77736}

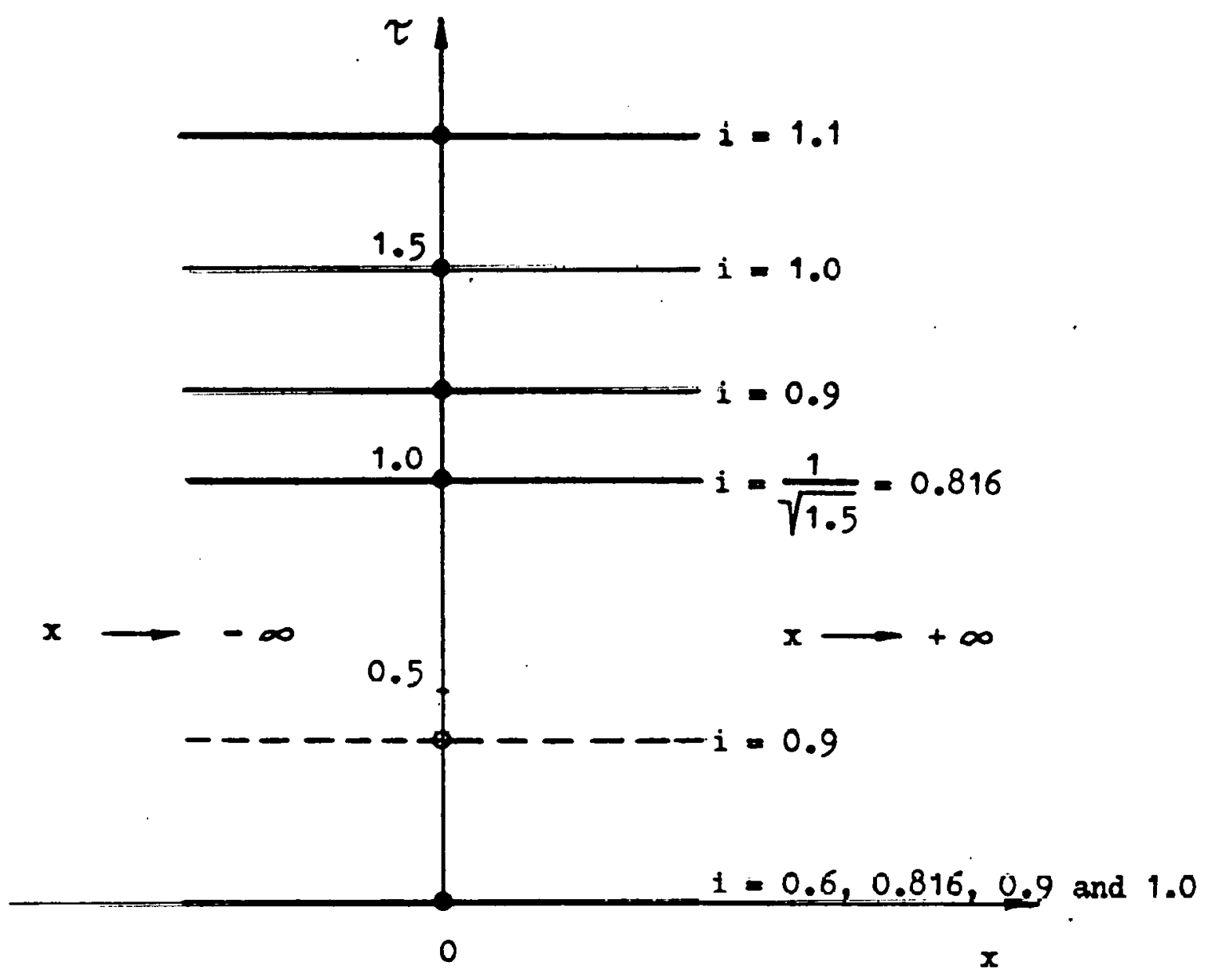

Fig. 3(a). $\tau(\mathrm{x})$ diagrams of stable and unstable equilibrium states for $\alpha=1.5$ (zero-dimensional analysis). 


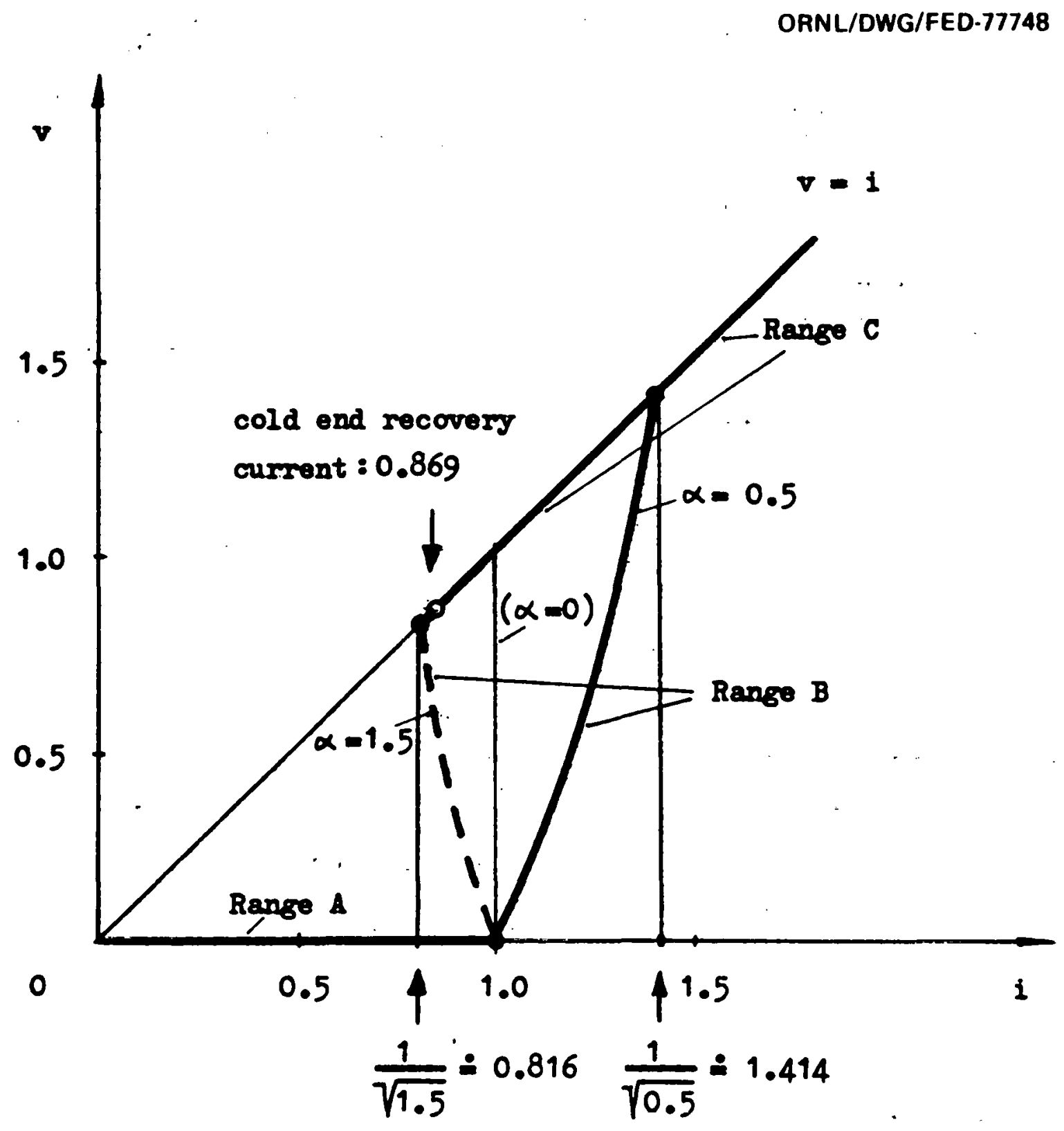

Fig. 4. v(i) characteristics for $\alpha=0.5$ and $\alpha=1.5$ assuming linear heat transfer (see Fig. 2 of Ref. 1). 
ORNL/DWG/FED-77750

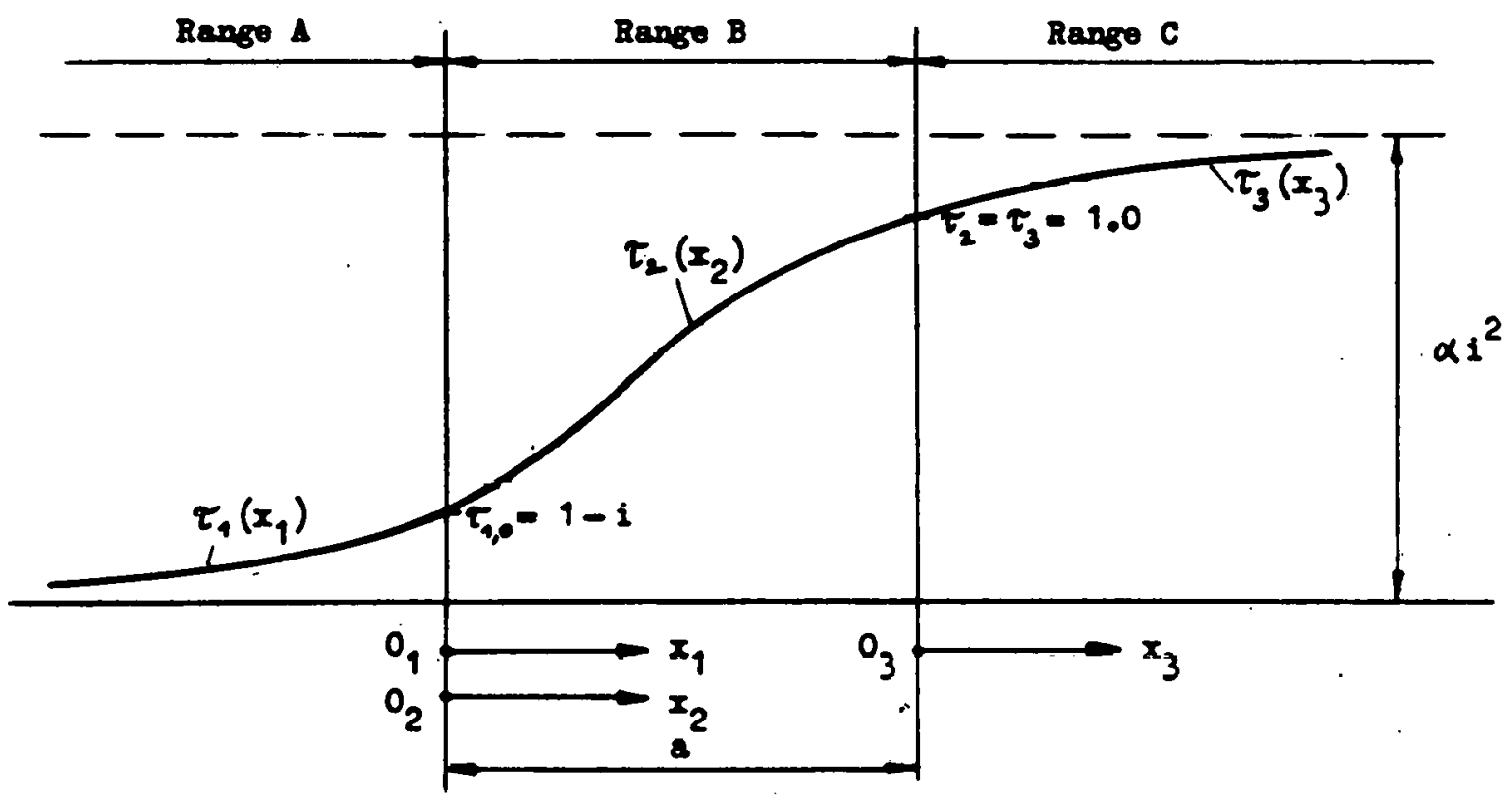

Fig. 5. Temperature profile of an infinitely long wire in the transition state of cold end recovery.

ORNLLDWG/FED.77749

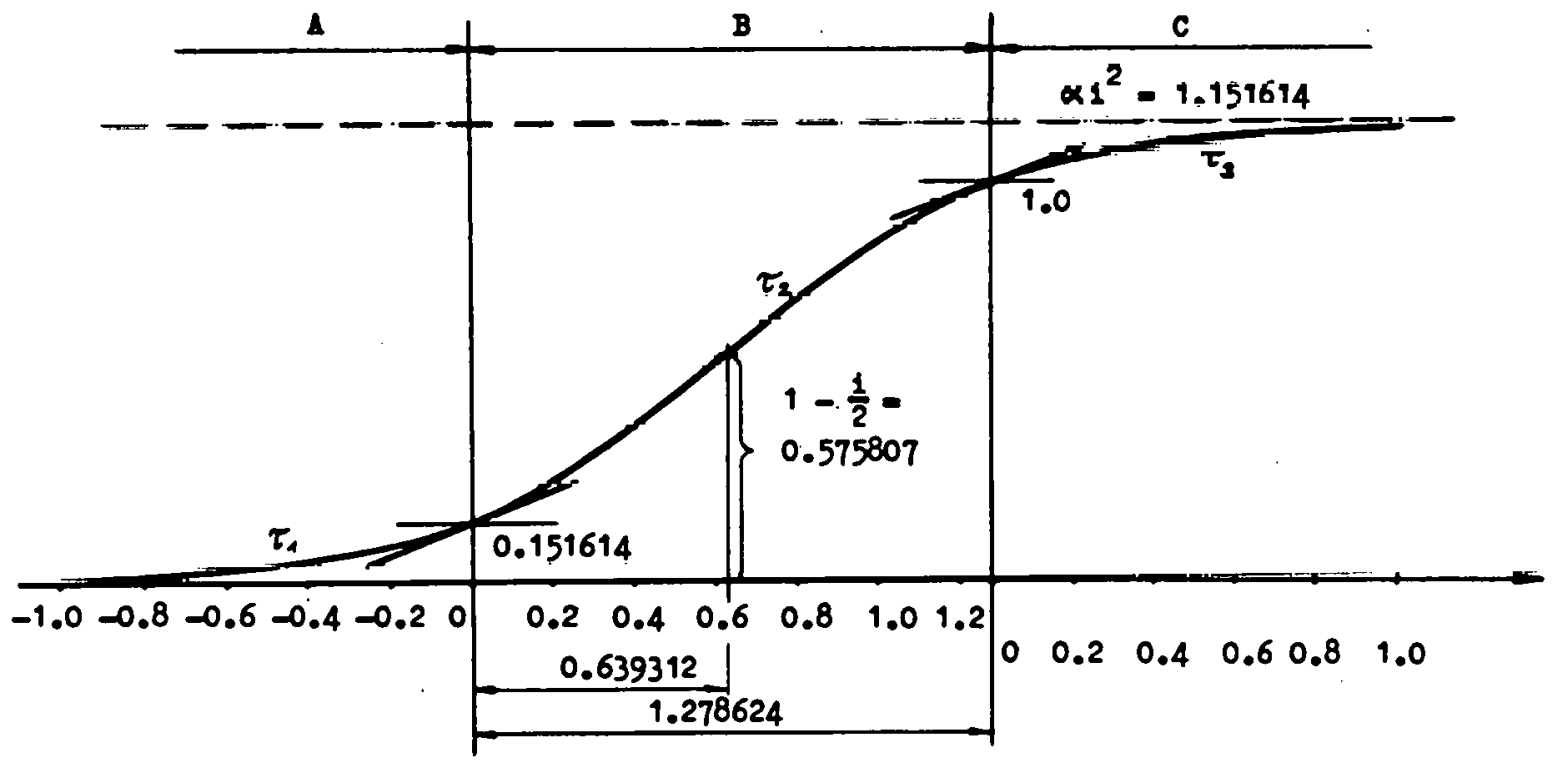

Fig. 6. Temperature profile of the transition state (cold end recovery) of a superconducting composite with $\alpha=1.6$ and $\beta=2.7$. 

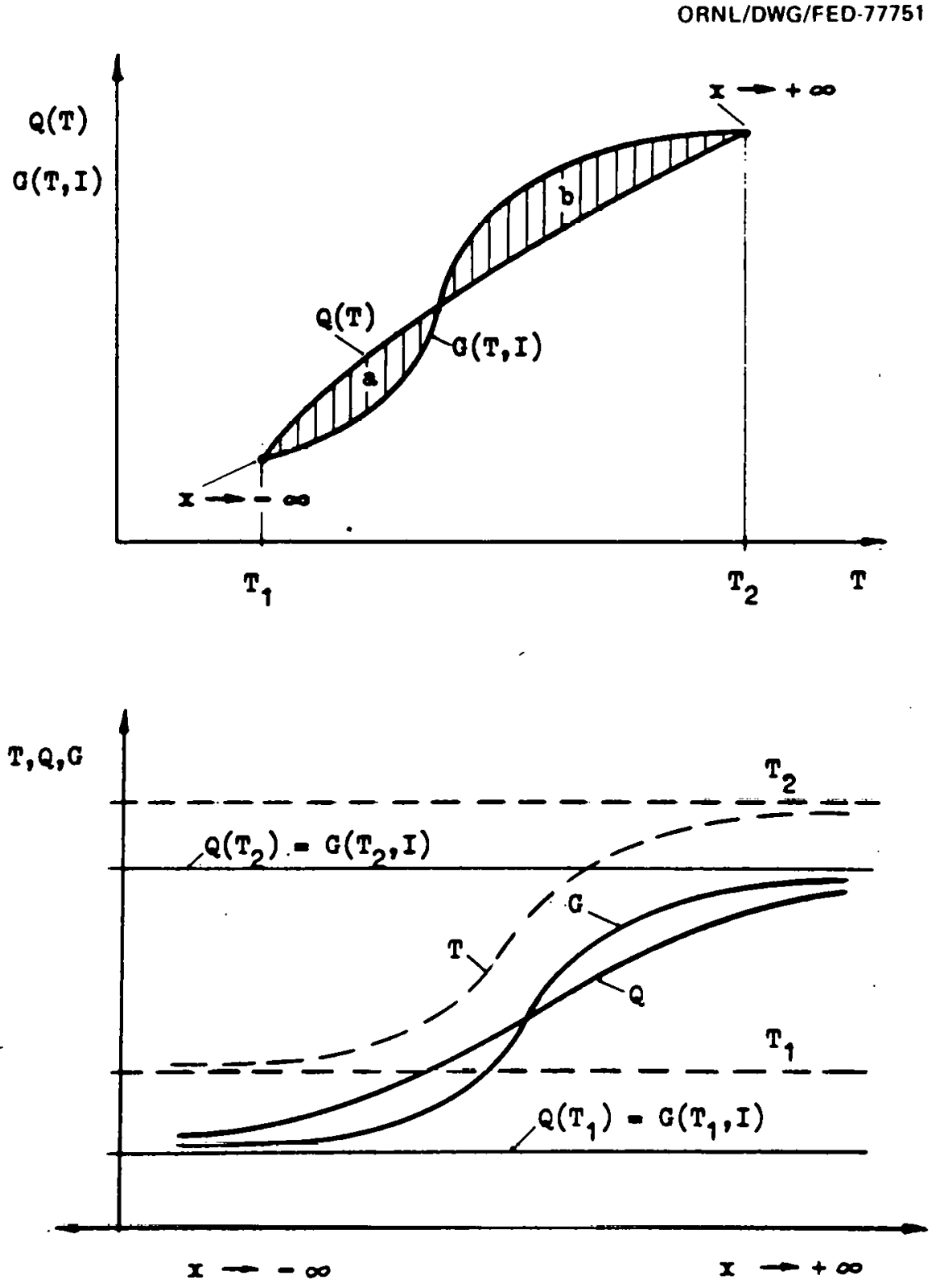

Fig. 7. Transitions between two zero-dimensional equilibrium states:

(a) rate of Joule heat production (G) and of heat loss (Q) as functions of the temperature $(T)$ and

(b) $G, Q$, and $T$ as functions of position along the superconducting composite. 
ORNL/DWG/FED-77753

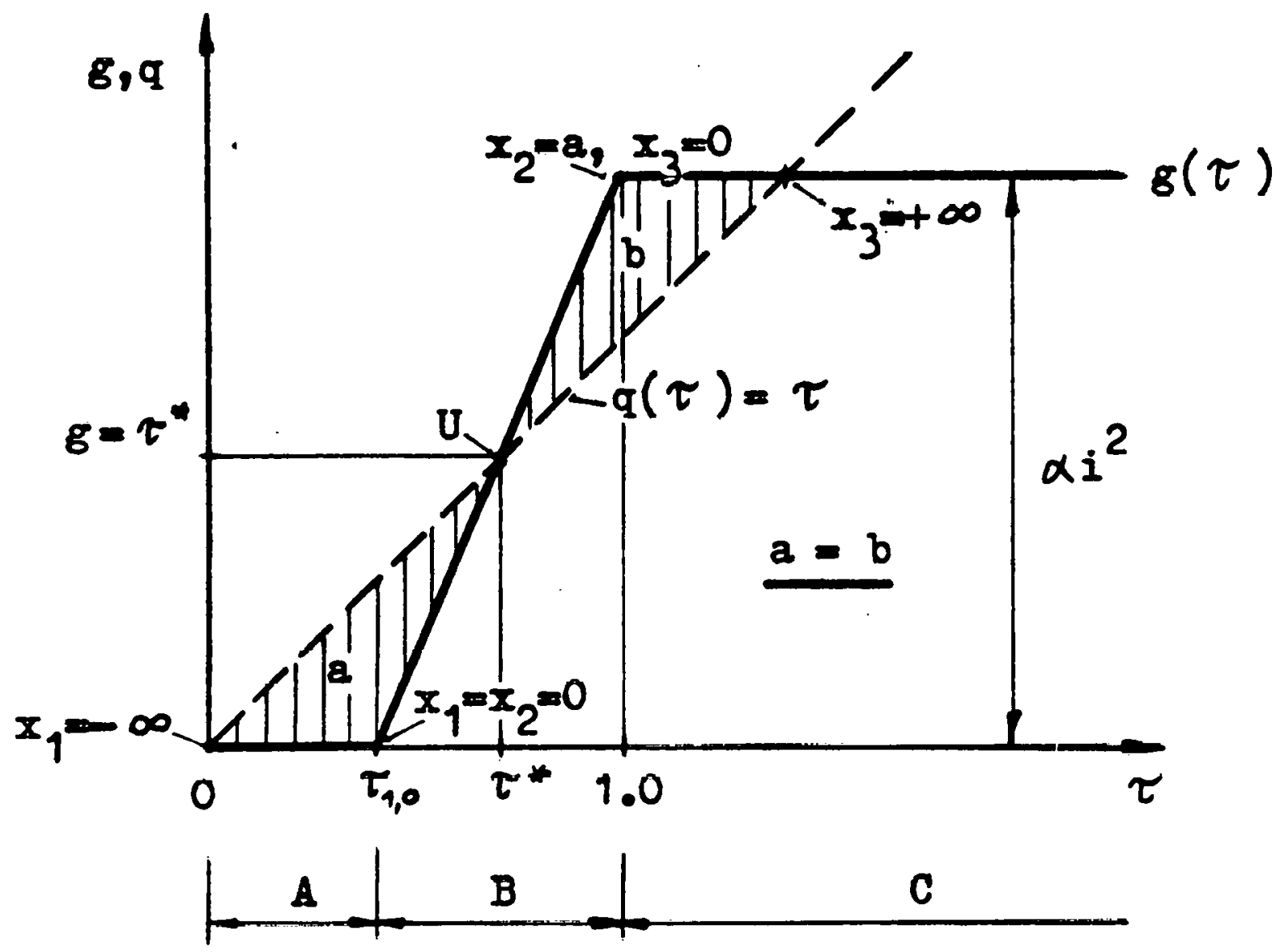

Fig. 8. $g(\tau)$ and $q(\tau)$ diagrams for the case of cold end recovery $(\alpha>1, \mathbf{i}<1)$. 


\section{ORNL/DWG/FED-77747}

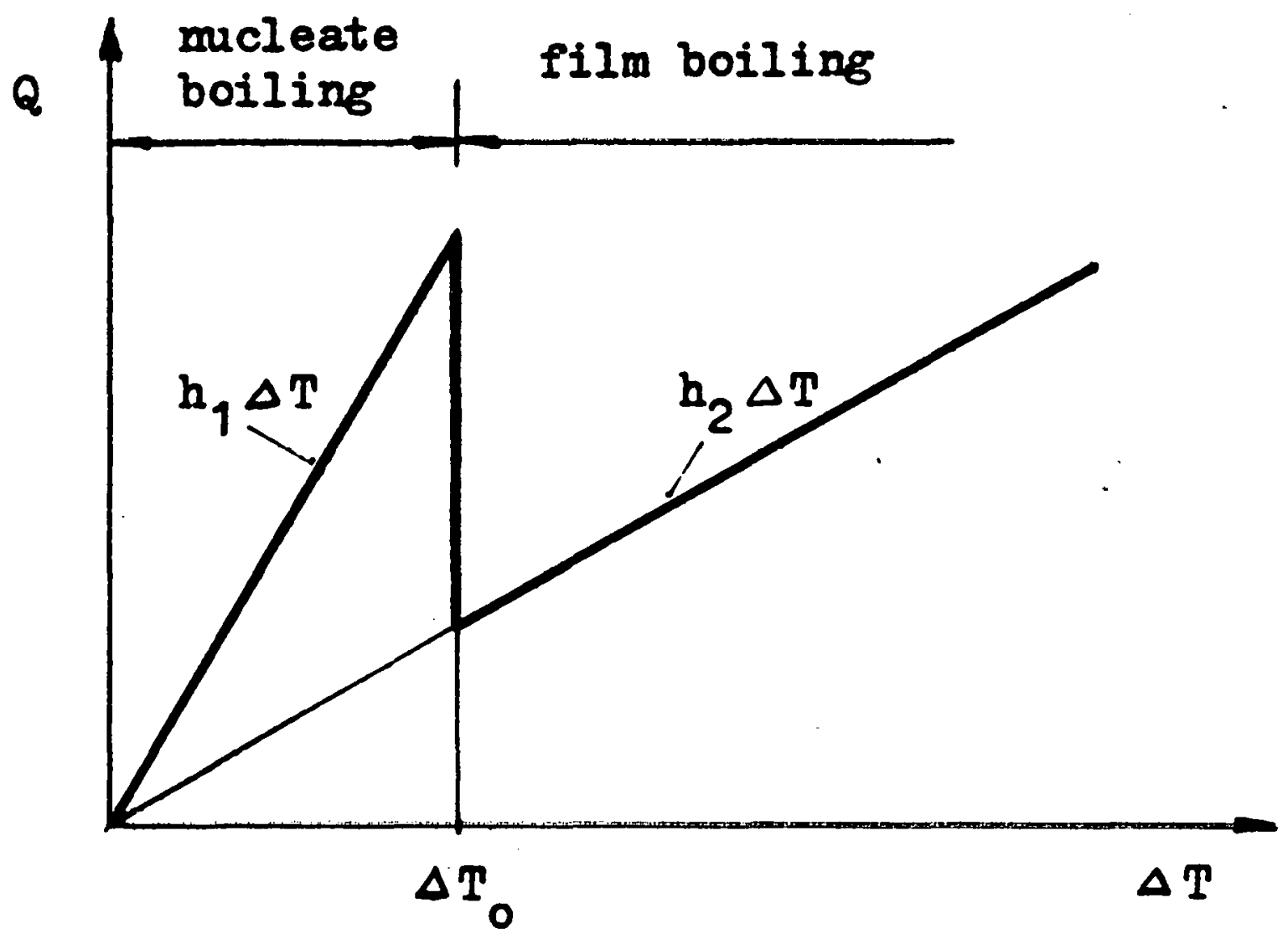

Fig. 9. $Q(\Delta T)$ characteristic similar to that shown by B. Montgomery (Ref. 7); (see Fig. I of this note). 


\section{ORNL/DWG/FED-77752}

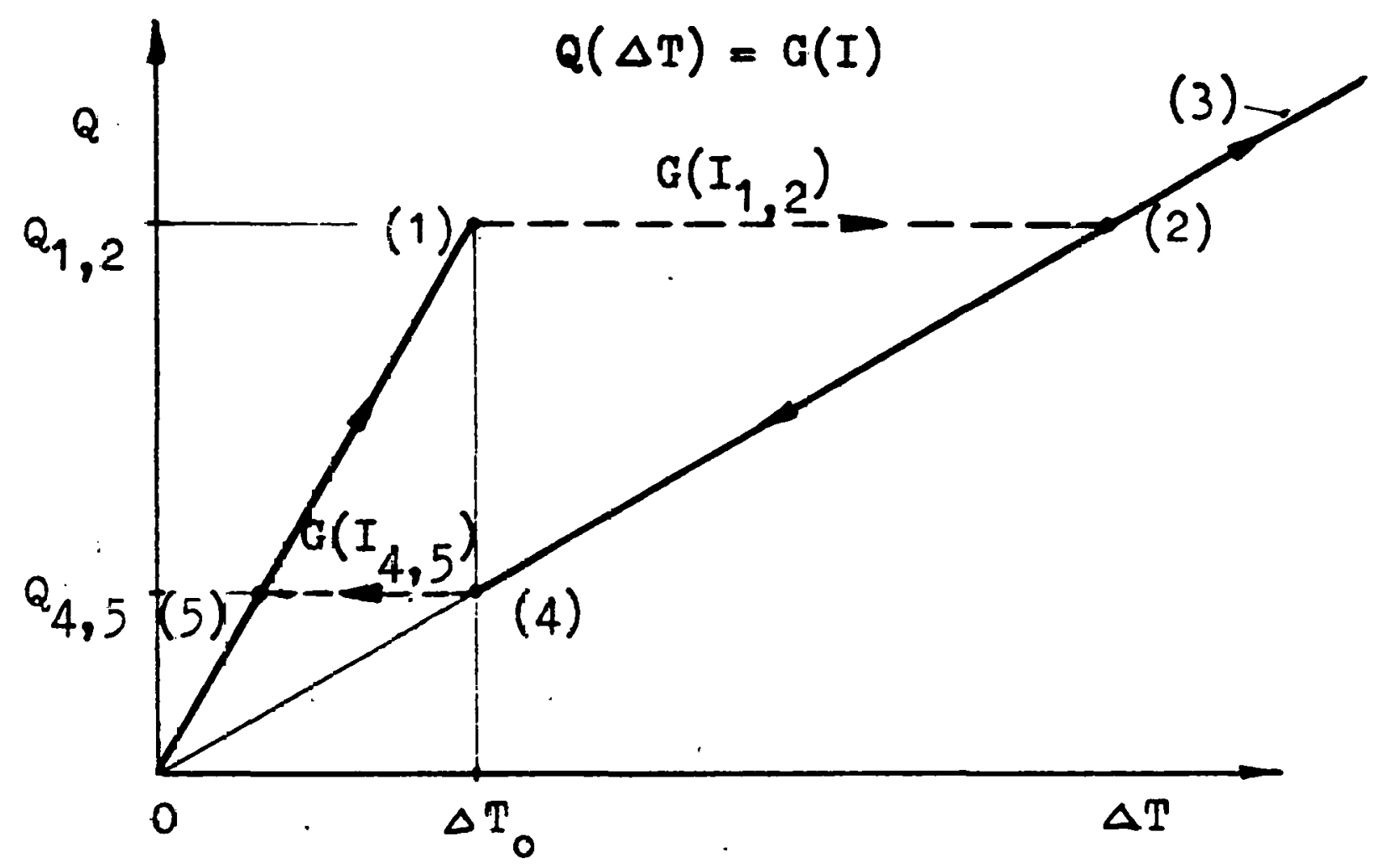

Fig. 10. Q(৫T) eycle for resistance wire with liquid heliun cooling.

$Z \in$ ro-dimensicnal analysis based on $Q(\Delta T)$ characteristic

shcwn in Fig. 9. 
ORNL/DWG/FED-77755

(a)

cooled an both onde (b)

Oooled on ase end Hoated on the of her end Cyole $(0),(7),(6),(3),(6),(7),(0)$ (o)

Heated on both ande Cycle $(0),(7),(6),(3),(4),(5),(0)$ cycle $(0),(1),(2),(3),(6),(7),(0)$
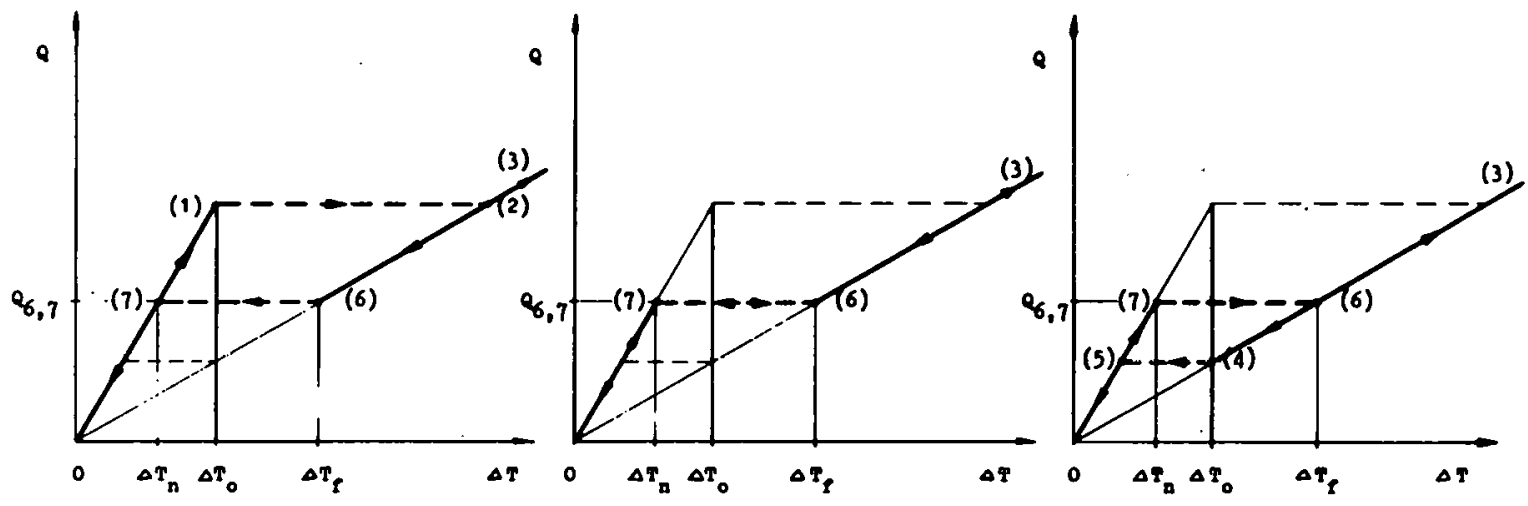

(Corrouponding teaperature profllee see Fig. 12)

Fig. 11. $Q(\Delta T)$ diagrams with various boundary conditions:

(a) cooled on both ends,

(b) cooled on one end and heated on the other end, and

(c) heated on both ends. 


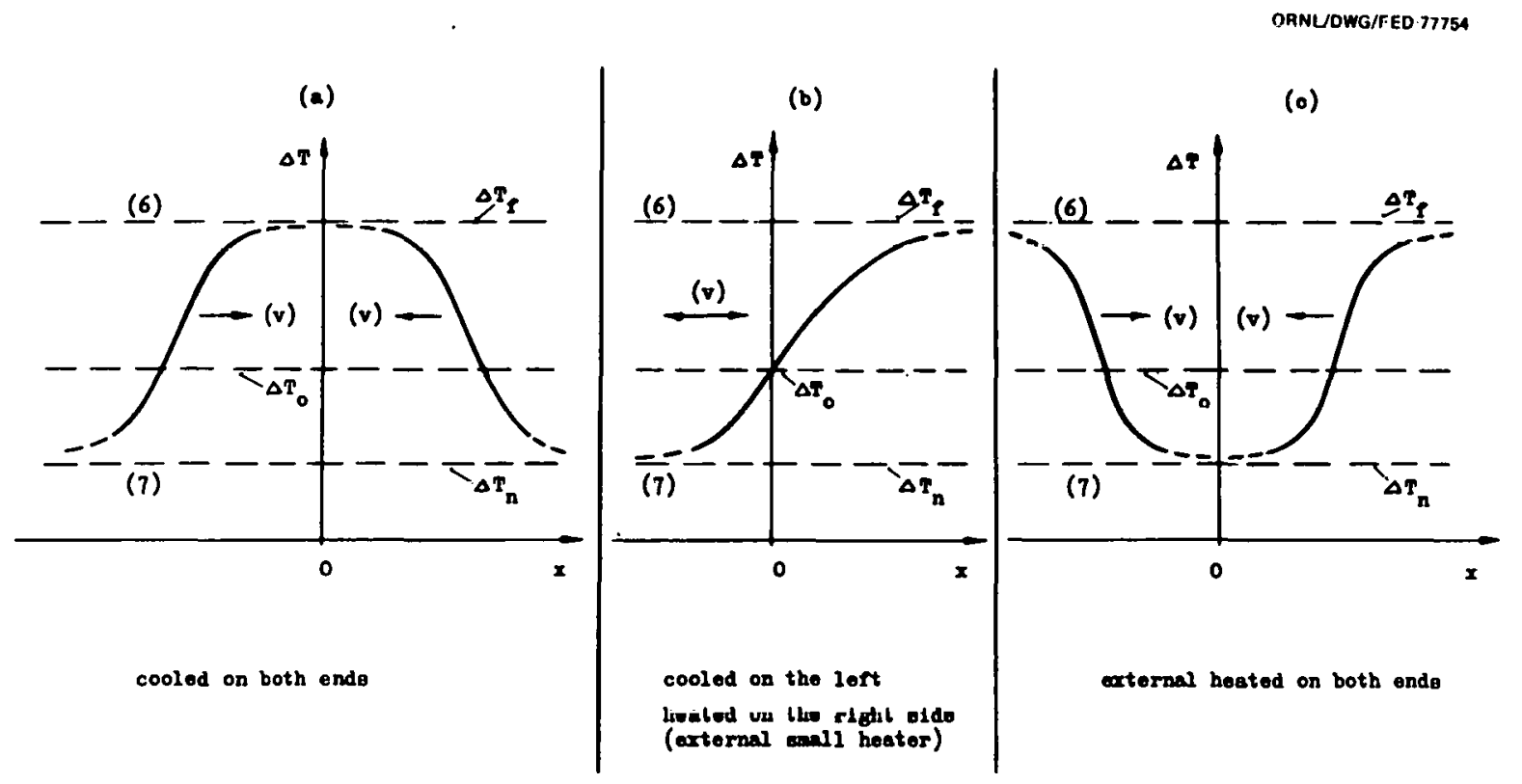

Fig. 12. Temperature profiles $[\Delta T(x)]$ of the helium-cooled resistance wire [transition zones (6) $\vec{t}(7)$ ] for various boundary conditions (schematical).

ORNL/OWG/FED-77741

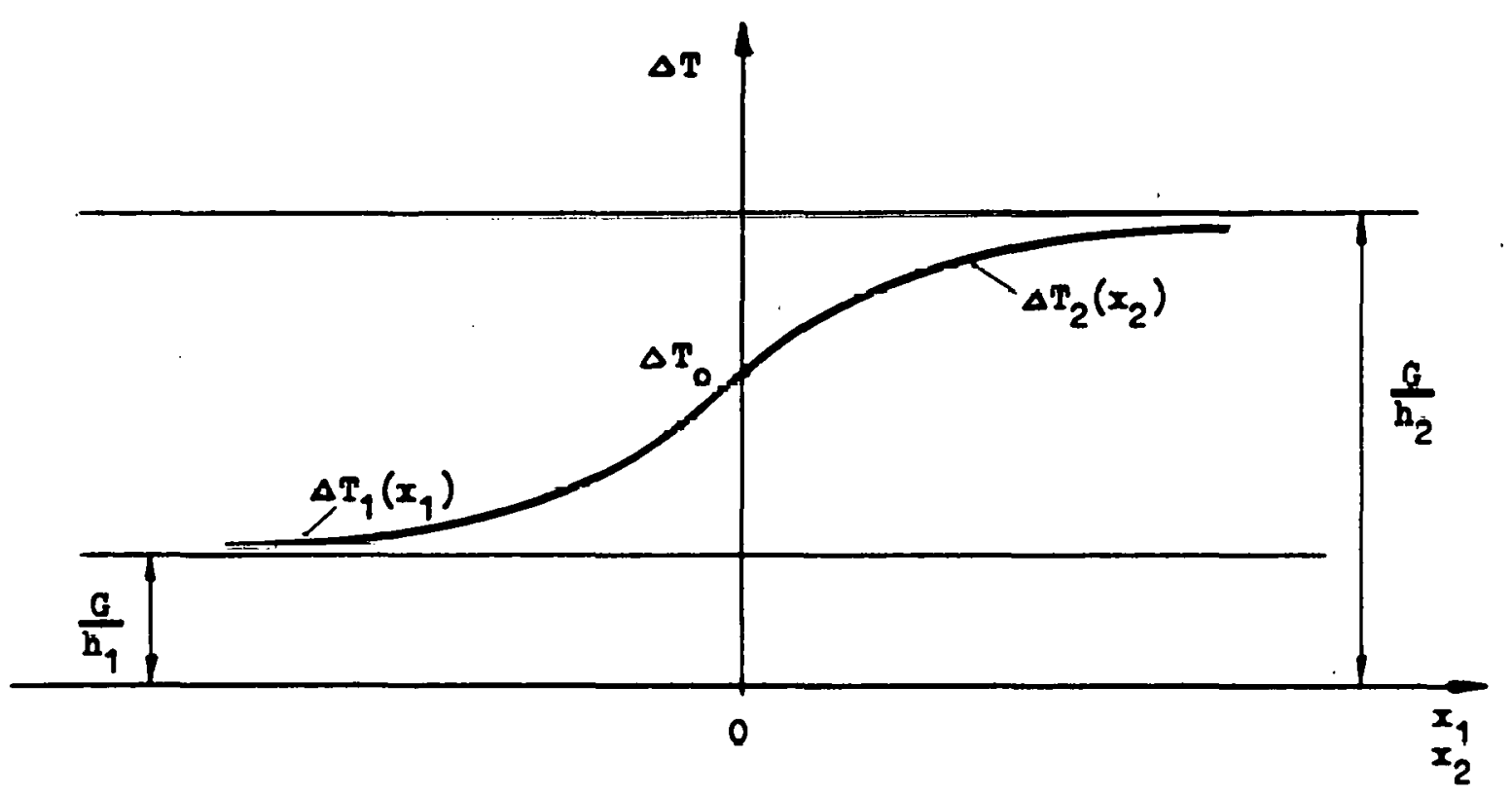

Fig. 13. $\Delta \mathrm{T}(\mathrm{x})$ diagram for transitions $(6) \rightleftarrows(7)$ (see Figs. 11 and 12 ). 
ORNL/DWG/FED-77742

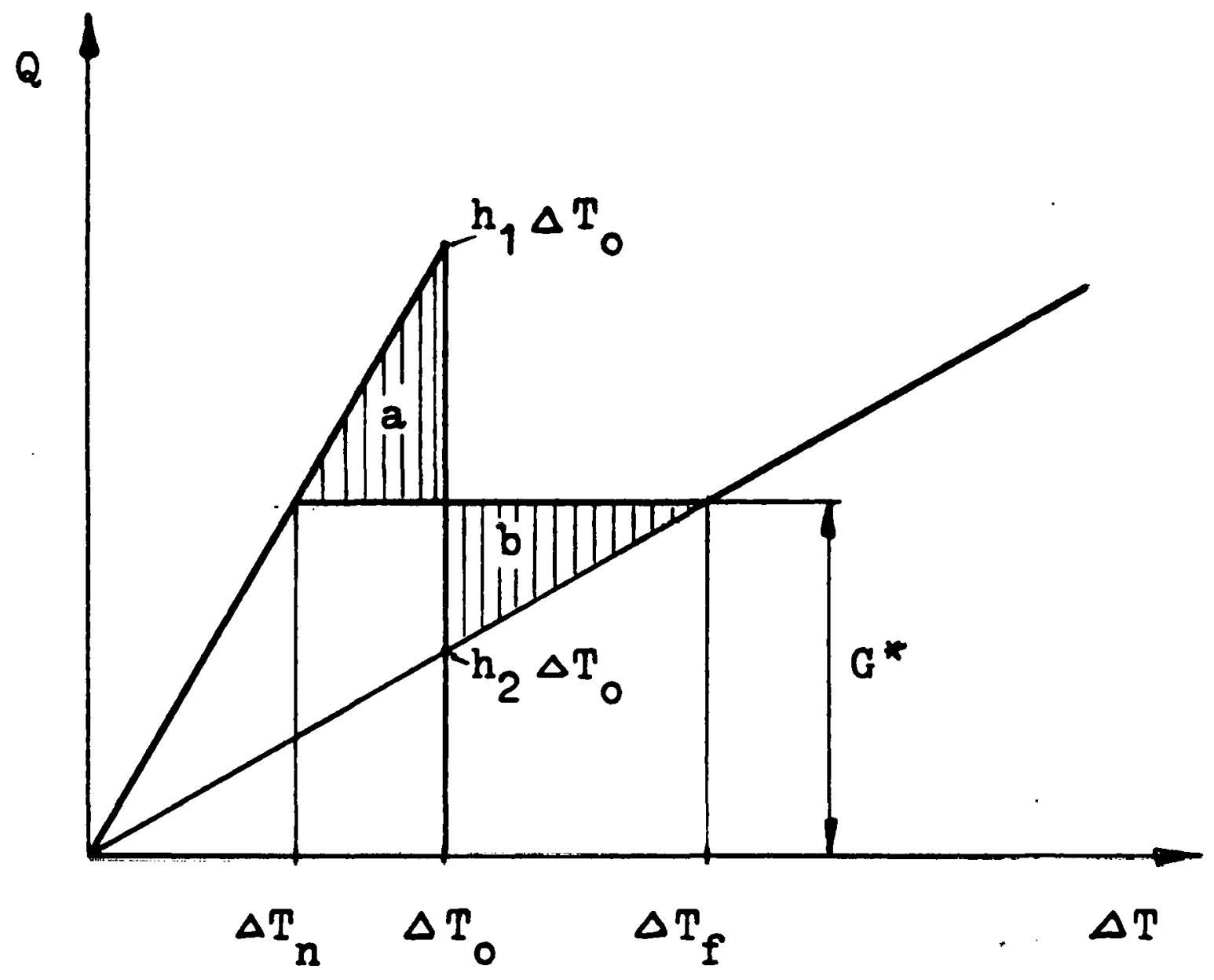

Fig. 14. Equal area conditions for a resistance wire with liquid helium cooling. 
ORNL/DWG/FED-77739

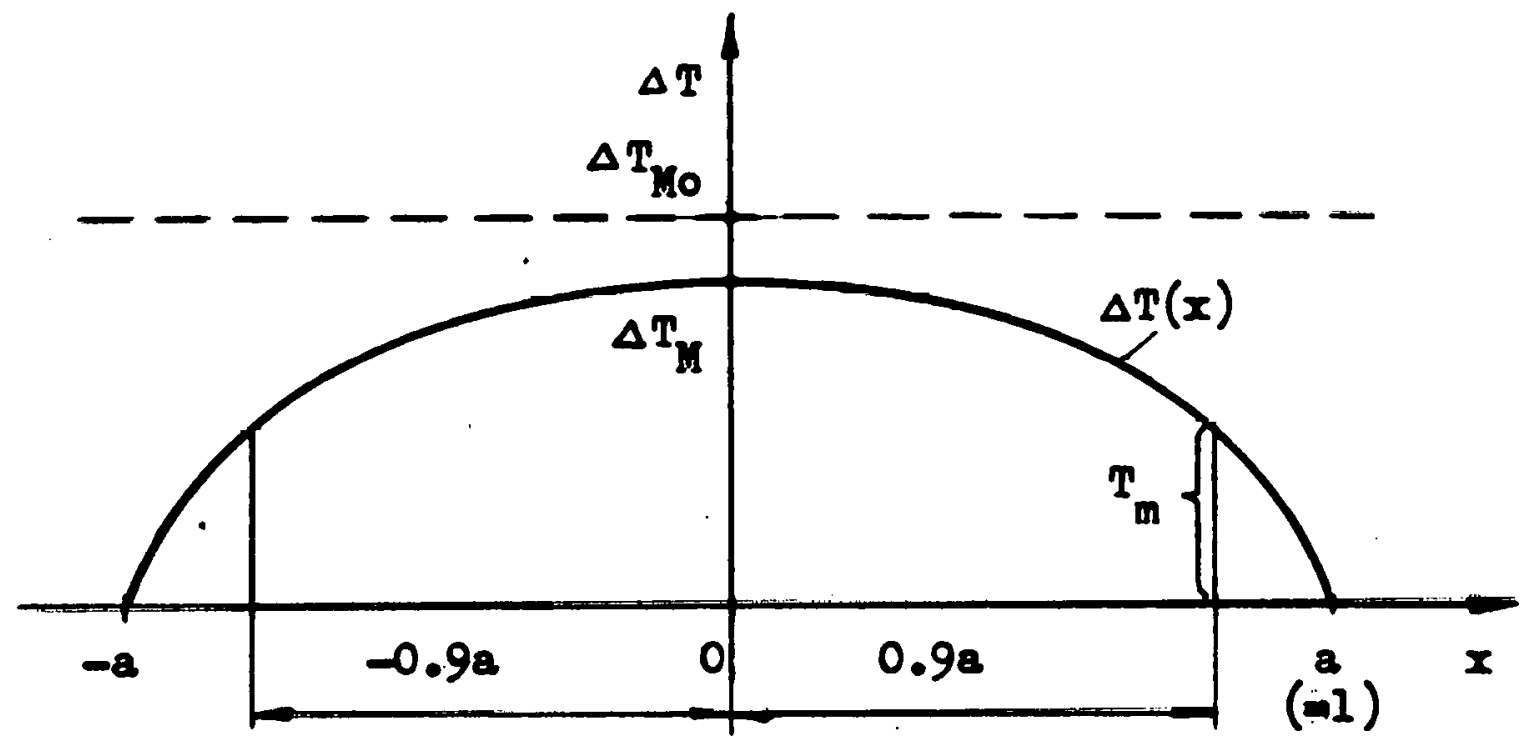

Fig. 15. Change of $\Delta T(x)$ inside a certain portion of the wire.

ORNL/DWG/FED-77738

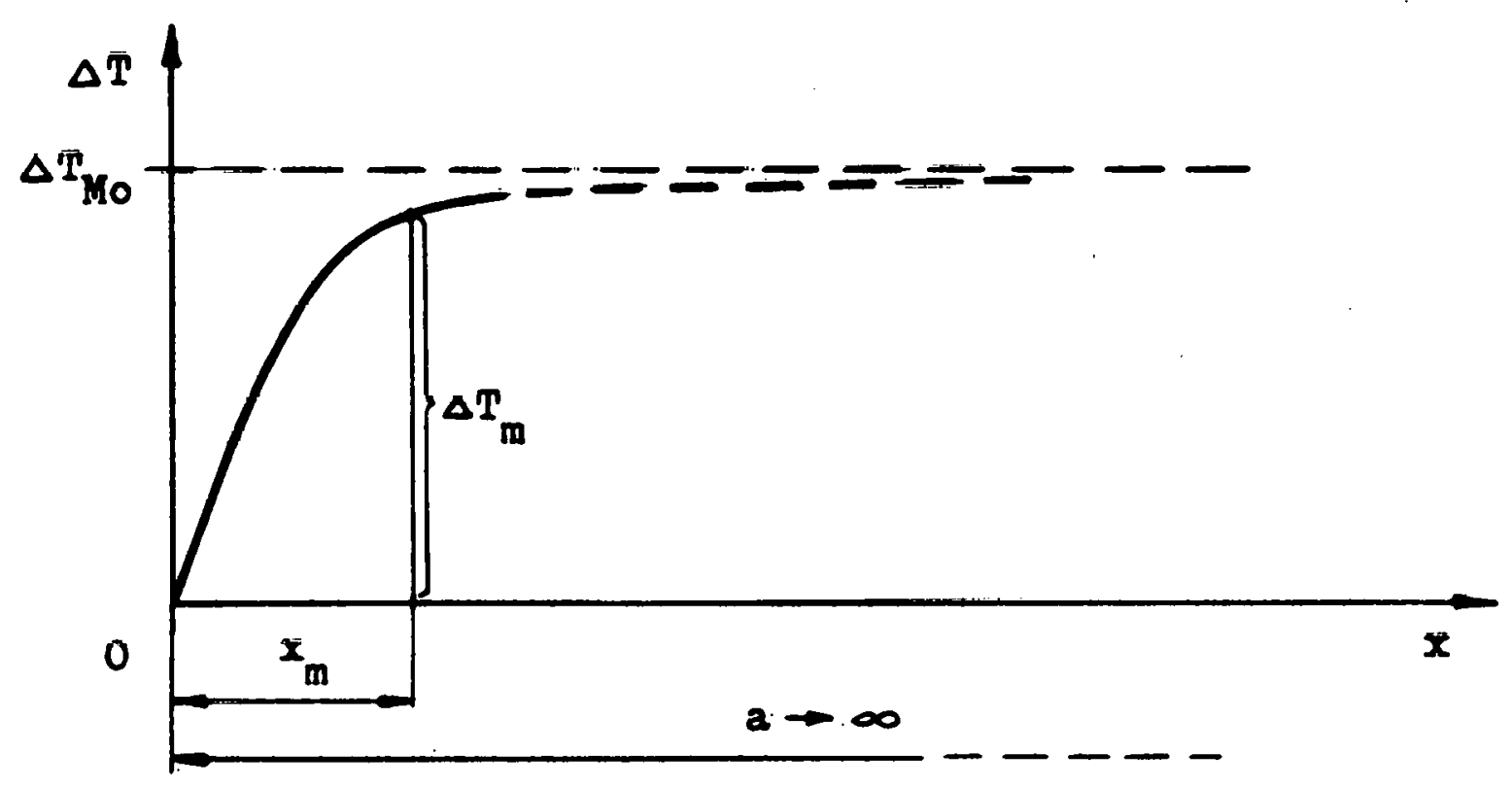

Fig. 16. $\Delta \mathrm{T}(\mathrm{x})$ near the terminal when $\mathrm{a} \rightarrow \infty$. 


\section{ORNL/DWG/FED-77740}

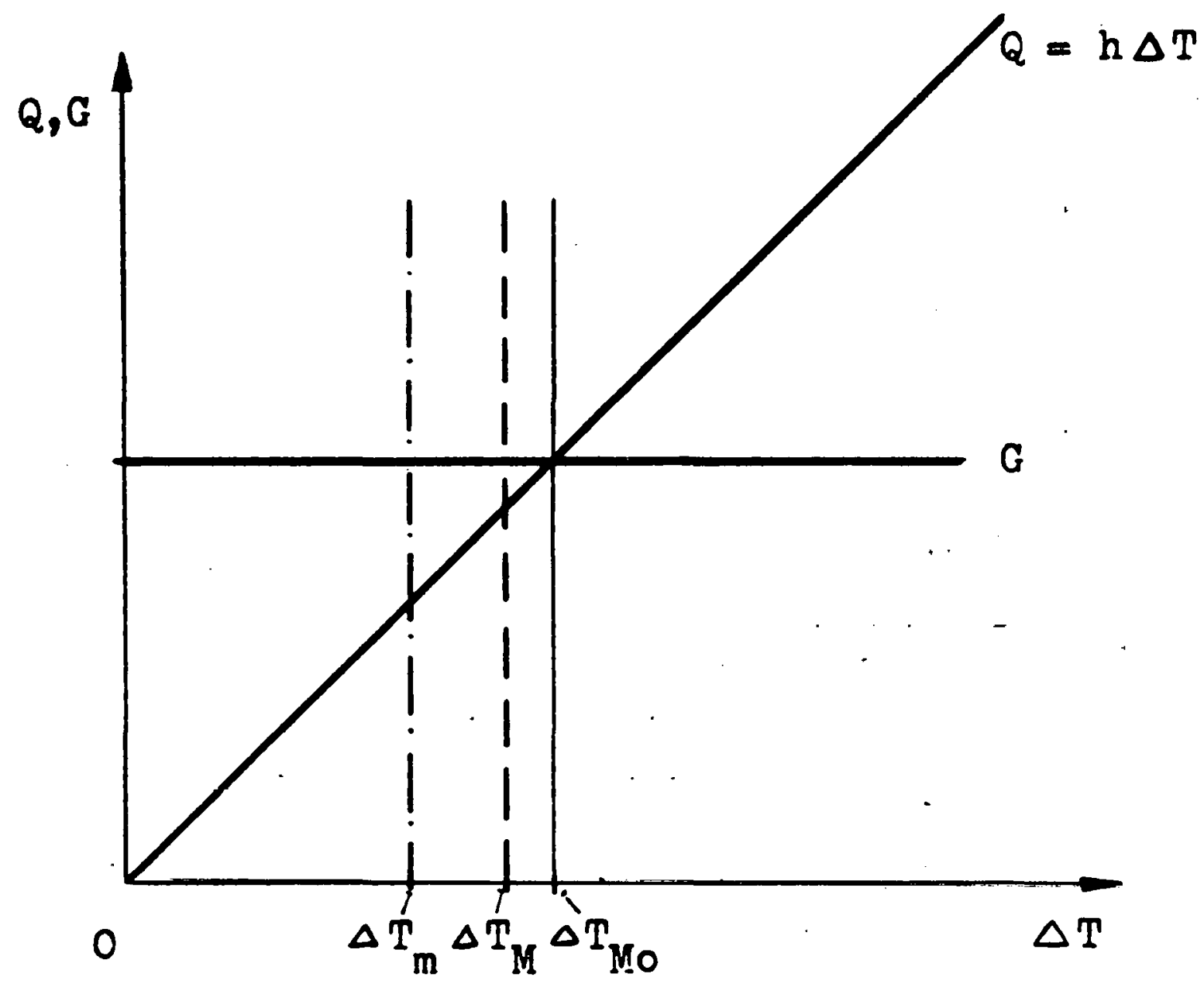

Fig. 17. Comparison between zero-dimensional solution $\Delta \mathrm{T}_{\mathrm{Mo}}$ and the values of $\Delta \mathrm{T}_{\mathrm{M}}$ and $\Delta \mathrm{T}_{\mathrm{m}}$ for a wire of finite length
(schematical). 
ORNL/DWG/FED-77734

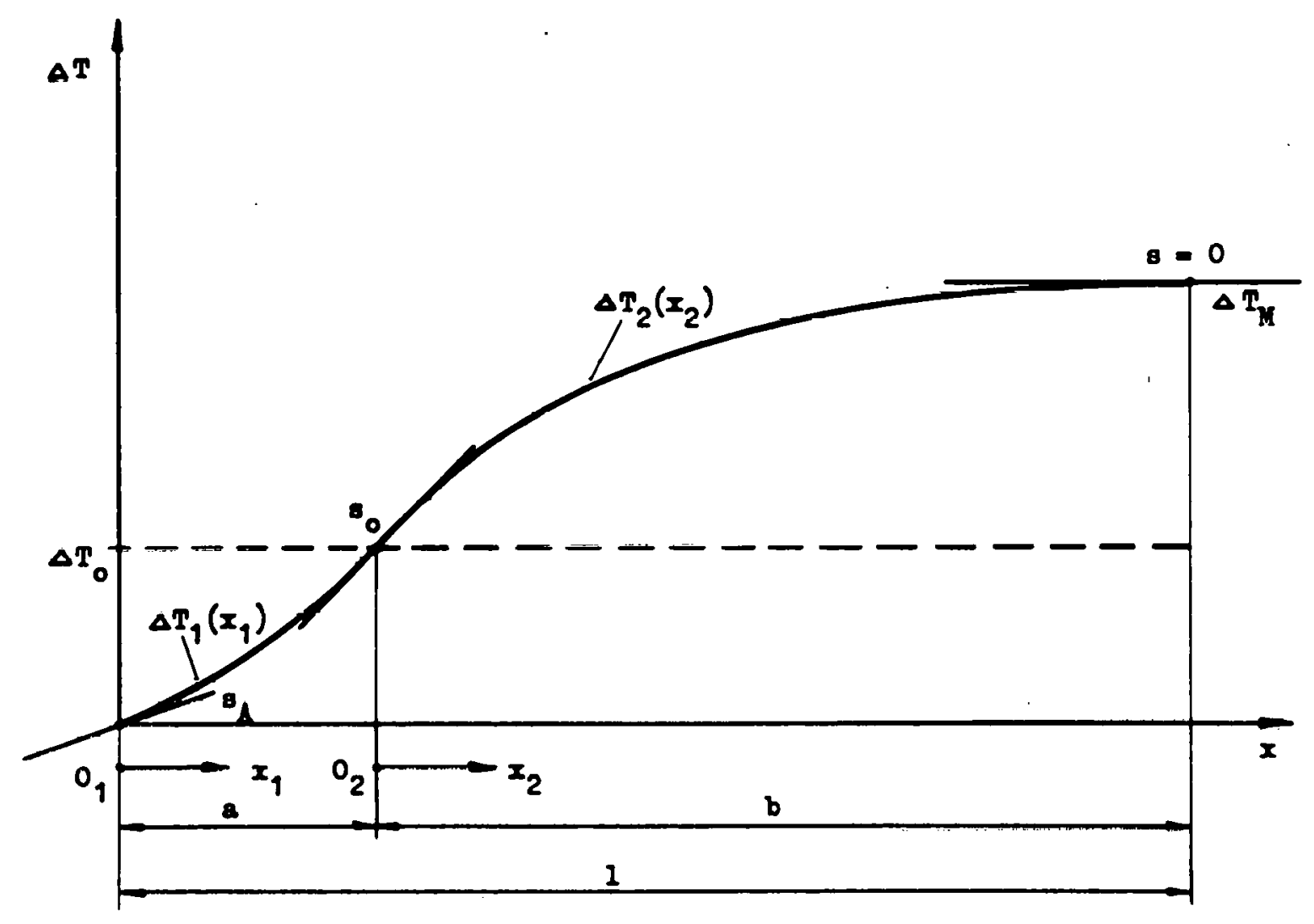

Fig. 18. Temperature profile $[\Delta T(x)]$ for the zone a of nucleate and for the zone $b$ of film boiling of the liquid helium (ochematical). 


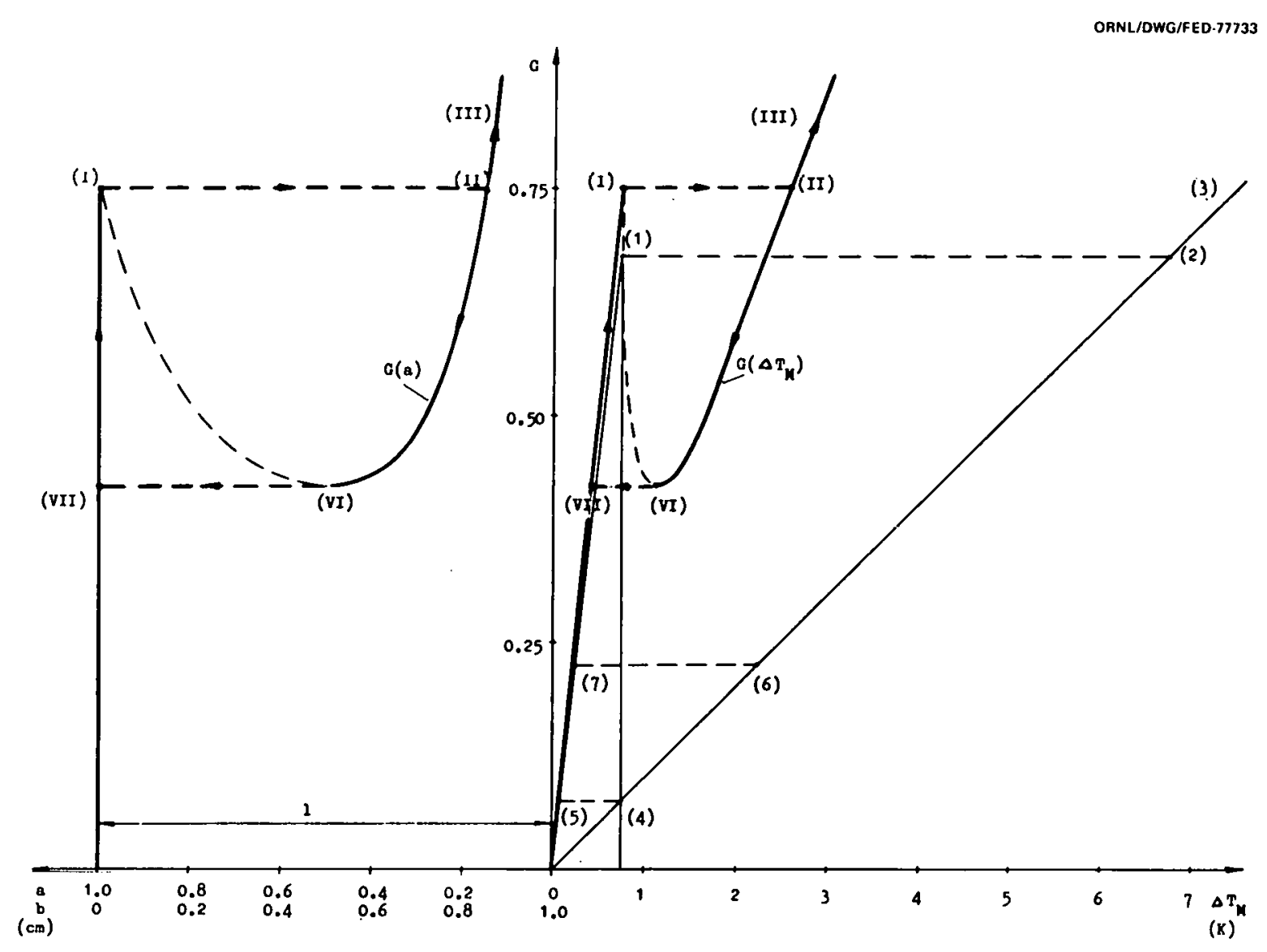

Fig. 19. $G(a)$ and $G\left(\Delta T_{M}\right)$ diagram for the resistance wire and a half-length of $\ell=1 \mathrm{~cm}$. 


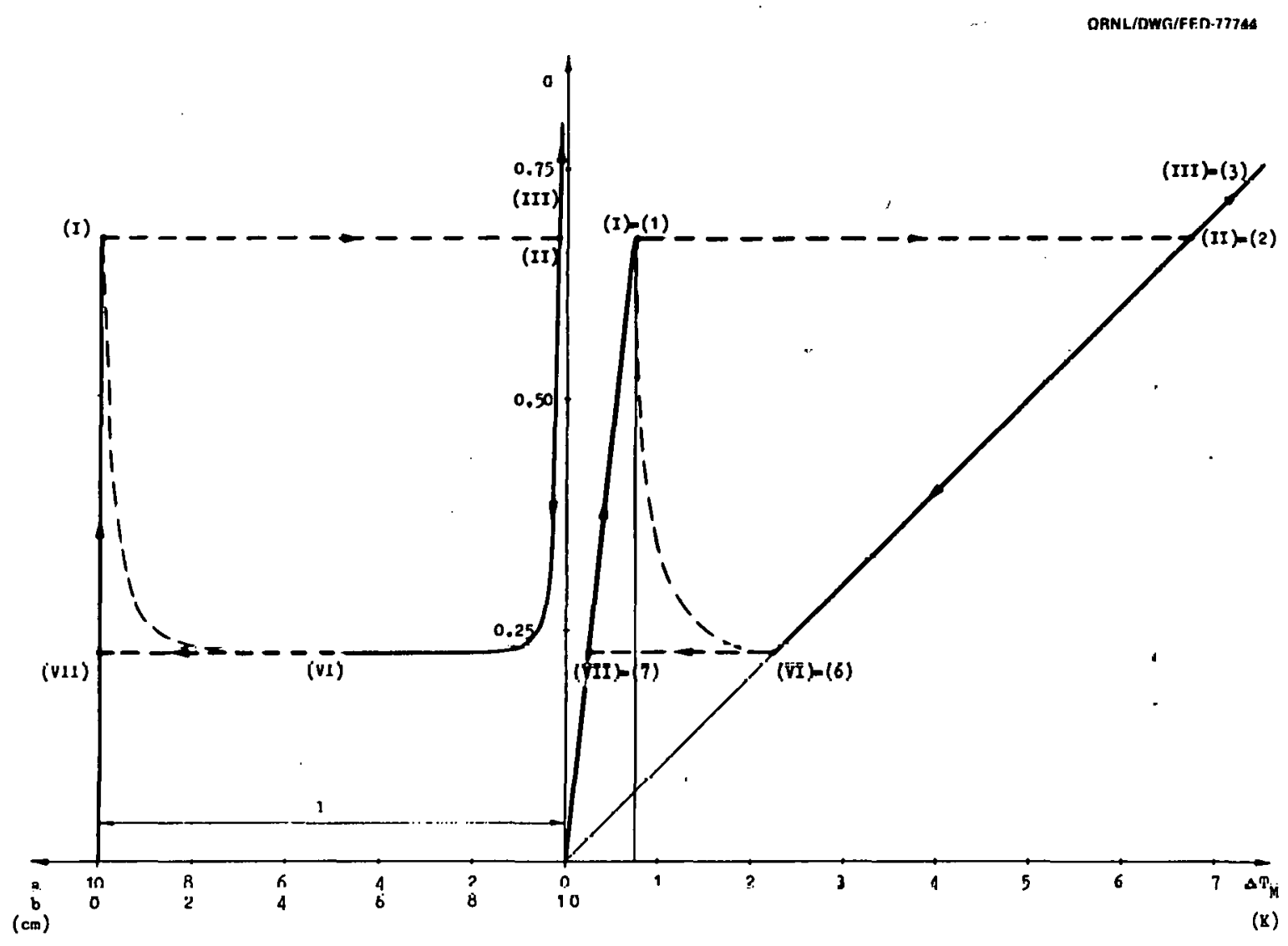

Fig. 20. $G(a)$ and $G\left(\Delta T_{M}\right)$ diagram for the wire with $\ell=10 \mathrm{~cm}$. 


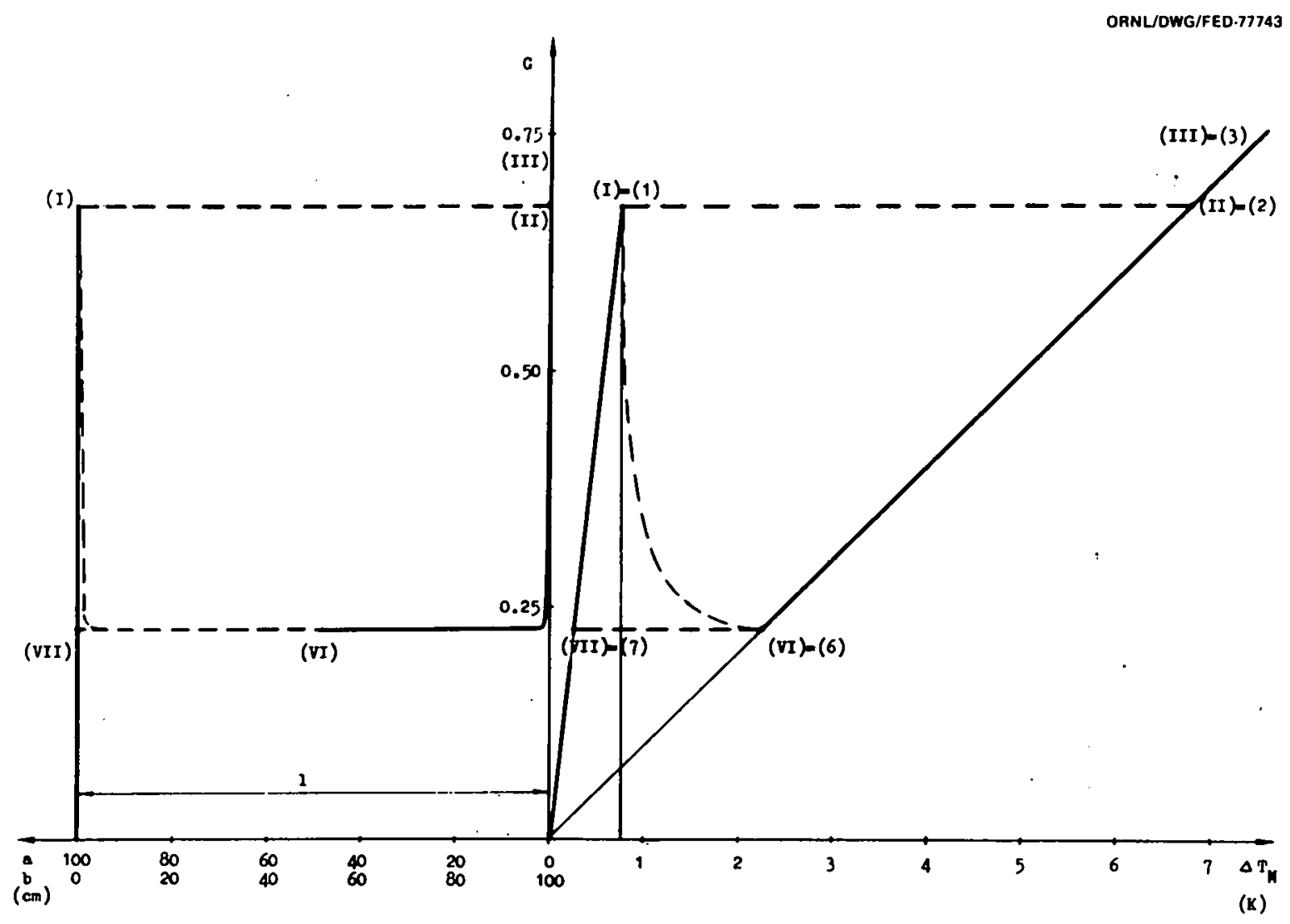

Fig. 21. $G(a)$ and $G\left(\Delta T_{M}\right)$ diagram for the wire with $\ell=100 \mathrm{~cm}$. 


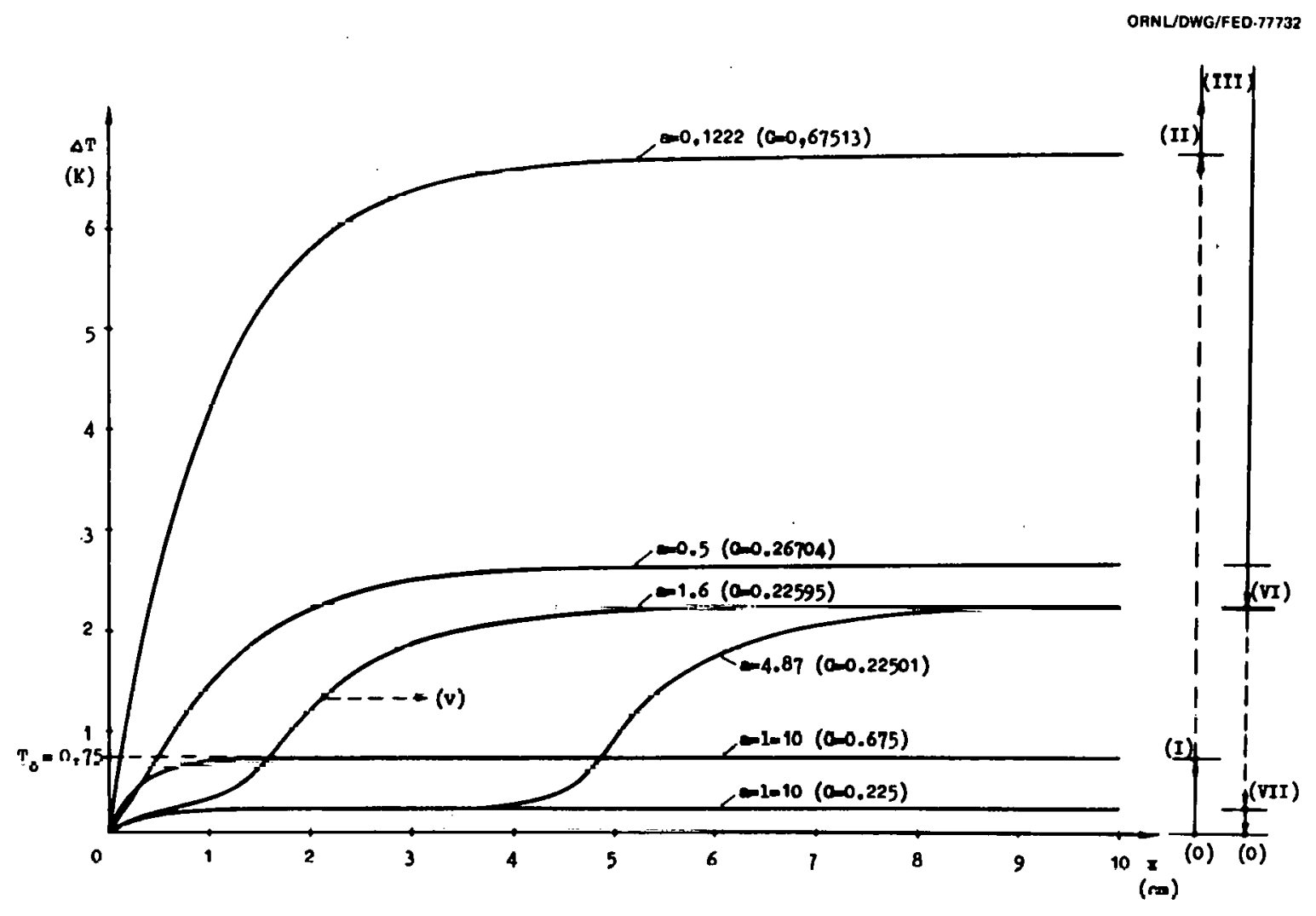

Fig. $22 \Delta T(x)$ diagram of the wire with $\ell=10 \mathrm{~cm}$. 


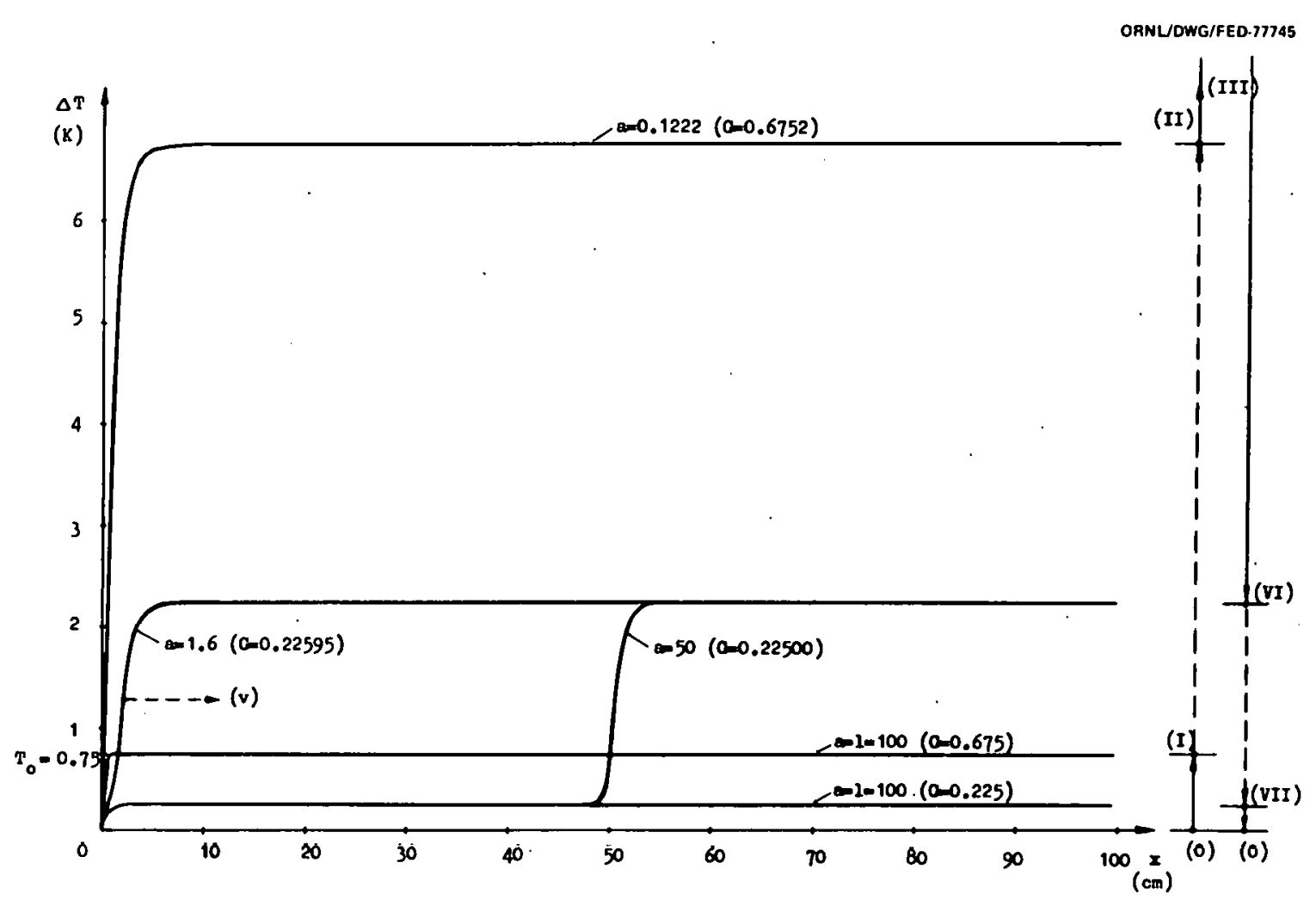

Fig. 23. $\Delta T(x)$ diagram of the wire with $\ell=100 \mathrm{~cm}$. 
THIS PAGE

\section{WAS INTENTIONALLY LEFT BLANK}




\section{REFERENCES}

1. Gauster, W. F., internal memorandum, 1975.

2. Stekly, 2.J.J., Proc. First Int. Cryog. Eng. Conf., Kyoto, 112 (1967) and many other publications, for example, Stekly et a1., NAS 8 - 2103 (August 1968).

3. Maddock, B. J., James, G. B., Norris, W. T., Cryogenics $\underline{9}, 261$ (1969).

4. Butler, A. P., James, G. B., Maddock, B. J., Norris, W. T., J. Heat Mass Transfer 13,105 (1970).

5. Refs. 7, 8, and 19 of Ref. (3).

6. Dresner, L., Cryogenics 16, 675 (1976).

7. Montgomery, D. B., "Superconducting Magnets - Where did we wrong?" Proc. 6th Symp. on Engineering Problems of Fusion Research, IEEE Pub. No. 75CH1097-5-NPS, Nov. 18-21, 1975.

8. Glossary of Terms in Superconduction (M. S. Lube1l, oral communications).

9. Williams, J.E.C., Phys. Lett. 19, 96 (1965).

10. Ljapunov, A. M., Stability of Motion, Acad. Press, New York and London, 1966. 


\section{THIS PAGE}

\section{WAS INTENTIONALLY LEFT BLANK}


ORNL/TM-5989

INTERNAL DISTRIBUTION

$\begin{aligned} \text { 1. } & \text { J. K. Ballou } \\ \text { 2. } & \text { R. L. Brown } \\ \text { 3. } & \text { P. B. Burn } \\ \text { 4. } & \text { L. Dresner } \\ \text { 5. } & \text { W. A. Fietz } \\ \text { 6. } & \text { W. H. Gray } \\ \text { 7. } & \text { P. N. Haubenreich } \\ \text { 8. } & \text { H. M. Long } \\ \text { 9. } & \text { J. C. Lottin } \\ \text { 10-21. } & \text { M. S. Lube11 } \\ 22 . & \text { J. W. Lue } \\ 23 . & \text { J. N. Luton } \\ 24 . & \text { J. R. Miller } \\ 25 . & \text { O. B. Morgan } \\ 26 . & \text { M. W. Rosenthal }\end{aligned}$

\author{
27. R. E. Schwall \\ 28. S. S. Shen \\ 29. W.C.T. Stoddart \\ 30. P. B. Thompson \\ 31. P. L. Walstrom \\ 32. II. T. Ych \\ 33-34. Central Research Library \\ 35. Fusion Energy Division \\ Communications office \\ 36. Document Reference Section \\ 37-38. Laboratory Records Department \\ 39. Laboratory Records - ORNL-RC. \\ 40. ORNL Patent office \\ 41-42. Fusion Energy Division Library
}

\section{EXTERNAL DISTRIBUTION}

43. E. Adam, Airco, 100 Mountain Avenue, Murray Hill, NJ 07974

44. V. D. Arp, National Bureau of Standards, Boulder, CO 80302

45. C. C. Baker, Fusion Power Program, Argonne National Laboratory, 9700 S. Cass Avenue, Argonne, IL 60439

46. R. W. Boom, 513 Engineering Research Building, University of Wisconsin, Madison, WI 53706

47. A. Clark, National Bureau of Standards, Boulder, Co 80302

48. D. L. Coffey, American Magnetics, Inc., P.0. Box R, Oak Ridge, TN 37830

49. D. N. Cornish, Lawrence Livermore Laboratory, P.0. Box 808, Livermore, CA 94550

50. D. A. Dingee, Manager, Fusion Programs, Battelle-Northwest, Battelle Boulevard, Richland, WA 99352

51. R. W. Fast, Manager, Experimental Facilities, National Accelerator Laboratory, P.0. Box 500, Batavia, IL 60510

52. J. J. Ferrante, General Electric Co., Schenectady, NY 12345

53. J. File, Princeton University, Plasma Physics Laboratory, Princeton, NJ 08540

54. D. S. Hackley, General Dynamics, Convair Division, P.0. Box 80847, San Diego, CA. 92138

55. W. V. Hassenzah1, Los Alamos Scientific Laboratory, P.0. Box 1663, Los Alamos, NM 87544

56. C. D. Henning, Division of Magnetic Fusion Energy, DOE, Mail Stop G-234, Washington, DC 20545

57. M. 0. Hoenig, Massachusetts Institute of Technology, National Magnet Laboratory, 170 Albany Street, Cambridge, MA 02139

58. C. K. Jones, Manager, Cryogenic Research Laboratory, Westinghouse Electric Corporation, Research and Development Center, Pittsburgh, PA 15235 
59. D. B. Montgomery, Massachusetts Institute of Technology, National Magnet Laboratory, 170 Albany Street, Cambridge, MA 02139

60. J. R. Powe11, Brookhaven Nationa1 Laboratory, Upton, NY 11073

61. J. R. Purcel1, General Atomic Company, P.0. Box 81608, San Diego, CA 92138

62. J. D. Rogers, Q-26, Los Alamos Scientific Laboratory, P.0. Box 1663, Los Alamos, NM 87544

63. C. H. Rosner, Intermagnetics General Corporation, Charles Industrial Park, New Karner Road, Guilderland, NY 12084

64. W. B. Sampson, Brookhaven National Laboratory, Upton, Long Island, NY 11973

65. Z.J.J. Stekly, Magnetic Corporation of America, 179 Bear Hill Road, Waltham, MA 02154

66. B. P. Strauss, Fermi National Accelerator Laboratory, P.0. Box 500, Batavia, IL 60510

67. C. von Keszycki, Grumman Aerospace Corporation, Research Center, Building 26, Bethpage, NY 11714

68. J. M. Willtams, Division of Magnetic Fusion Energy, DOE, Mail Stop G-234, Washington, DC 20545

69. S. L. Wipf, Q-26, Los Alamos Scientific Research Laboratory, P.0. Box 1663, Los Alamos, NM 87544

70. E. J. Ziurys, Division of Magnetic Fusion Energy, DOE, Mail Stop G-234, Washington, DC 20545

71. Director, Research and Technical Support Division, Department of Energy, Oak Ridge Operations, P.0. Box E, Oak Ridge, TN 37830

72-98. Technical Information Center, P.0. Box 62, Oak Ridge, TN 37830 